\title{
Excitation of Kerr quasinormal modes in extreme-mass-ratio inspirals
}

\author{
Jonathan Thornburg, ${ }^{1,2}$ Barry Wardell $\odot,{ }^{3}$ and Maarten van de Meent $\oplus^{2,4}$ \\ ${ }^{1}$ Department of Astronomy and Center for Spacetime Symmetries, Indiana University, Bloomington, Indiana 47405, USA \\ ${ }^{2}$ Max-Planck-Institut für Gravitationsphysik, Albert-Einstein-Institut, Am Mühlenberg 1, D-14476 Potsdam-Golm, Germany \\ ${ }^{3}$ School of Mathematics and Statistics, University College Dublin, Belfield, Dublin 4, DO4 V1W8, Ireland \\ ${ }^{4}$ Mathematical Sciences and STAG Research Centre, University of Southampton, Southampton SO17 1BJ, United Kingdom
}

(Received 10 July 2019; accepted 12 November 2019; published 25 March 2020)

\begin{abstract}
If a small compact object orbits a black hole, it is known that it can excite the black hole's quasinormal modes (QNMs), leading to high-frequency oscillations ("wiggles") in the radiated field at $\mathcal{J}^{+}$, and in the radiation-reaction self-force acting on the object after its orbit passes through periapsis. Here we survey the phenomenology of these wiggles across a range of black hole spins and equatorial orbits. In both the scalar-field and gravitational cases, we find that wiggles are a generic feature across a wide range of parameter space, and they are observable in field perturbations at fixed spatial positions, in the self-force, and in radiated fields at $\mathcal{J}^{+}$. For a given charge or mass of the small body, the QNM excitations have the highest amplitudes for systems with a highly spinning central black hole, a prograde orbit with high eccentricity, and an orbital periapsis close to the light ring. However, the QNM amplitudes remain nonzero for all black hole spins and for retrograde as well as for prograde orbits. The QNM amplitudes depend smoothly on the orbital parameters, with only very small amplitude changes when the orbit (discrete) frequency spectrum is tuned to match QNM frequencies. The association of wiggles with QNM excitations suggests that they represent a situation where the nonlocal nature of the self-force is particularly apparent, with the wiggles arising as a result of QNM excitation by the compact object near periapsis, and then encountered later in the orbit. Astrophysically, the effects of wiggles at $\mathcal{J}^{+}$might allow direct observation of Kerr QNMs in extreme-mass-ratio inspiral (EMRI) binary black hole systems, potentially enabling new tests of general relativity.
\end{abstract}

DOI: 10.1103/PhysRevResearch.2.013365

\section{INTRODUCTION}

Consider a small (compact) body of mass $\mu M$ (with $0<$ $\mu \ll 1$ ) moving freely near a Schwarzschild or Kerr black hole of mass $M$. This system emits gravitational radiation, and there is a corresponding radiation-reaction influence on the small body's motion. Calculating the resulting perturbed spacetime (including the small body's motion and the emitted gravitational radiation) is a long-standing research question in general relativity.

There is also an astrophysical motivation for this calculation: If a neutron star or stellar-mass black hole of mass $\sim 1-$ $100 M_{\odot}$ orbits a massive black hole of mass $\sim 10^{5}-10^{7} M_{\odot},{ }^{1}$ the resulting "extreme-mass-ratio inspiral" (EMRI) system is expected to be a strong astrophysical gravitational-wave (GW) source detectable by the planned Laser Interferometer Space Antenna (LISA) space-based gravitational-wave detector. LISA is expected to observe many such systems, some of them at quite high signal-to-noise ratios [1-4]. The data analysis for, and indeed the detection of, such systems will

Published by the American Physical Society under the terms of the Creative Commons Attribution 4.0 International license. Further distribution of this work must maintain attribution to the author(s) and the published article's title, journal citation, and DOI.

${ }^{1} M_{\odot}$ denotes the solar mass. generally require matched-filtering the detector data stream against appropriate precomputed GW templates. The problem of computing such templates provides an astrophysical motivation for EMRI modeling.

In the test-particle limit, it has long been known that an unbound (scattering) flyby can excite quasinormal modes (QNMs) of the background black hole. Kojima and Nakamura [5] studied this process, finding that "A scattered particle excites the quasinormal mode under the condition that twice the angular velocity at the periapsis is greater than the real part of the frequency of the quasinormal mode." Their Fig. 3(b) shows an example of the QNM oscillations in the radiated gravitational waves at $\mathcal{J}^{+}$.

Burko and Khanna [6] found small oscillations in the total radiated energy flux from a test particle making a parabolic (unbound) flyby of a Kerr black hole. They attributed these oscillations to the particle encountering scattered gravitational waves emitted during the particle's inbound motion.

O'Sullivan and Hughes [7] observed "small-amplitude high-frequency oscillations" in their calculations of the horizon shear of a Kerr black hole orbited by a test particle. Because they did not find corresponding oscillations in the horizon tidal distortion field, and their measured oscillation frequencies did not match known Kerr QNM frequencies, they concluded that the horizon-shear oscillations they observed "cannot be related to the [Kerr black] hole's quasinormal modes." 
Thornburg and Wardell [8] (hereinafter TW) calculated the scalar-field self-force for eccentric equatorial particle orbits in Kerr spacetime. For some systems where the Kerr black hole was highly spinning and the particle orbit was prograde and highly eccentric, TW found that the self-force exhibits large oscillations ("wiggles") on the outgoing leg of the orbit shortly after periapsis passage. TW suggested that wiggles "are in some way caused by the particle's close passage by the large black hole." Thornburg $[9,10]$ presented fits of dampedsinusoid models to these wiggles for a range of Kerr spins and particle orbits, found close agreement of the model's complex frequencies with those of known Kerr QNMs, and argued that this agreement shows that wiggles are, in fact, caused by Kerr QNMs excited by the particle's close periapsis passage.

Yang et al. [11] studied binary neutron-star systems with close periapses and highly eccentric orbits, finding that neutron-star vibrational modes are excited by the close flyby and radiate enough GWs to significantly influence the orbital evolution.

Nasipak, Osburn, and Evans [12] calculated the scalar-field self-force and the radiated field at $\mathcal{J}^{+}$for eccentric inclined particle orbits in Kerr spacetime. For one particular (equatorial) orbit configuration they confirmed TW's finding of wiggles in the self-force and also found wiggles in the radiated scalar field at $\mathcal{J}^{+}$, fitting these to a superposition of $\ell=m=1$, $\ell=m=2, \ell=m=3$, and $\ell=m=4$ Kerr quasinormal modes (QNMs). They concluded that wiggles are caused by Kerr QNMs excited by the particle's close periapsis passage.

Rifat, Khanna, and Burko [13] recently studied wiggles for EMRIs where the central Kerr BH is nearly extremal (dimensionless spin up to 0.99999), finding that in such systems many Kerr QNMs can be simultaneously excited.

Here we extend Refs. $[9,10]$ and survey wiggles' phenomenology over a wide range in parameter space for eccentric equatorial orbits in Kerr space-time, for both the scalar-field model and the full gravitational field. We focus on leading-order radiation and radiation-reaction effects computed via 1st-order perturbations of Kerr space-time, i.e., (for the gravitational case) $\mathcal{O}(\mu)$ field perturbations near the Kerr black hole, $\mathcal{O}(\mu)$ radiation at $\mathcal{J}^{+}$, and $\mathcal{O}\left(\mu^{2}\right)$ radiationreaction "self-forces" acting on the small body. We fit models of Kerr QNMs to all these diagnostics.

Our focus is the case where the perturbing body's orbit is highly relativistic, so post-Newtonian methods (see, for example, Sec. 6.10 in Ref. [14] and Refs. [15-20] and references therein) are not reliably accurate. Since the timescale for radiation reaction to shrink the orbit is very long $\left(\sim \mu^{-1} M\right)$, while the required resolution near the small body is very high $(\sim \mu M)$, a direct "numerical relativity" integration of the Einstein equations (see, for example, Refs. [21-25] and references therein) would be prohibitively expensive (and probably insufficiently accurate) for this problem. ${ }^{2}$ Instead,

\footnotetext{
${ }^{2} \mathrm{~A}$ number of researchers have attempted direct numericalrelativity binary black hole simulations for systems with "intermediate" mass ratios up to $100: 1(\mu=0.01)$, (see, for example, Refs. [26-32]). However, it has not (yet) been possible to extend these results to the extreme-mass-ratio case nor to accurately evolve
}

we use black hole perturbation theory, treating the small body as an $\mathcal{O}(\mu)$ perturbation on the background spacetime.

We present results obtained from two separate numerical codes which were programed independently, using completely different theoretical formalisms and numerical methods:

(1) Our scalar-field results were obtained using TW's code $[8,34]$ extended to compute additional field diagnostics. This code uses an effective-source regularization followed by an $e^{i m \phi}$ Fourier-mode decomposition and a separate $2+1$ dimensional time-domain numerical evolution of each Fourier mode. The main outputs of this code are the regularized scalar field at selected (fixed) spatial positions, the regularized scalar field and self-force at the particle, and the physical radiated scalar field at $\mathcal{J}^{+}$.

(2) Our gravitational-field results were obtained using van de Meent's code [35-38]. This code obtains the local metric perturbation in the frequency domain by reconstructing the metric perturbation from the Weyl scalar $\Psi_{4}$, followed by $\ell$-mode regularization to obtain the regular part. The main outputs of this code are the regularized outgoing-radiationgauge metric perturbation and self-force at the particle, and the physical radiated $\Psi_{4}$ at $\mathcal{J}^{+}$and selected fixed positions in the spacetime.

For both codes we take the particle orbit to be a bound equatorial geodesic; we do not consider changes in the orbit induced by the self-force.

To briefly summarize our main results, we observe wiggles across a wide range of Kerr spins and particle orbits. Wiggles are present in all of our field diagnostics in the strongfield region and at $\mathcal{J}^{+}$. Except for a few anomalous results for near-extremal Kerr spacetimes (dimensionless Kerr spins $\gtrsim 0.9999$ ), our results are all consistent with the interpretation of wiggles as Kerr QNMs. Wiggles are stronger and more readily observable for high Kerr spins, highly eccentric prograde particle orbits, and particle periapsis radii close to the light ring.

The remainder of this paper is organized as follows. Section I A summarizes our notation and our parametrization of bound geodesic orbits in Kerr spacetime. Section II briefly summarizes our calculations of scalar-field (Sec. II A) and gravitational (Sec. II B) perturbations of Kerr spacetime. Section III describes our local- and radiated-field diagnostics. Section IV describes our QNM models and how we fit these to time series of our field diagnostics. Section $\mathrm{V}$ presents our data for scalar-field and gravitational perturbations of Kerr spacetime, and QNM-model fits to this data. Finally, Sec. VI discusses our results and presents our conclusions.

\section{A. Notation and parametrization of Kerr geodesics}

We generally follow the sign and notation conventions of Wald [39], with $G=c=1$ units and a $(-,+,+,+)$ metric signature. We use the Penrose abstract-index notation, with indices abcd running over spacetime coordinates, and $i j k$ running over the spatial coordinates. $\nabla_{a}$ is the (spacetime)

any systems with mass ratios more extreme than 18:1 for a radiationreaction timescale [32] (Hinder [33] reports ongoing efforts to extend this to $32: 1$ ). 
covariant derivative operator and $g$ is the determinant of the spacetime metric. $X:=Y$ means that $X$ is defined to be $Y$. $\square:=\nabla_{a} \nabla^{a}$ is the four-dimensional (scalar) wave operator $[40,41]$.

We use Boyer-Lindquist coordinates $(t, r, \theta, \phi)$ on Kerr spacetime, defined by the line element

$$
\begin{aligned}
d s^{2}= & -\left(1-\frac{2 M r}{\Sigma}\right) d t^{2}-4 M^{2} \tilde{a} \frac{r \sin ^{2} \theta}{\Sigma} d t d \phi \\
& +\frac{\Sigma}{\Delta} d r^{2}+\Sigma d \theta^{2} \\
& +\left(r^{2}+M^{2} \tilde{a}^{2}+2 M^{3} \tilde{a}^{2} \frac{r \sin ^{2} \theta}{\Sigma}\right) \sin ^{2} \theta d \phi^{2},
\end{aligned}
$$

where $M$ is the black hole mass, $\tilde{a}=J / M^{2}$ is the black hole dimensionless spin (limited to $|\tilde{a}|<1), \Sigma:=r^{2}+M^{2} \tilde{a}^{2} \cos ^{2} \theta$, and $\Delta:=r^{2}-2 M r+M^{2} \tilde{a}^{2}$. We also define $a:=M \tilde{a}$ (this is unrelated to the use of $a$ as an abstract tensor index). In these coordinates the event horizon is the coordinate sphere $r=r_{+}=M\left(1+\sqrt{1-\tilde{a}^{2}}\right)$ and the inner horizon is the coordinate sphere $r=r_{-}=M\left(1-\sqrt{1-\tilde{a}^{2}}\right.$ ). (See footnote 3 for a discussion of TW's coordinate compactification near the event horizon and $\mathcal{J}^{+}$.)

Following Ref. [42], we define the tortoise coordinate $r_{*}$ by

$$
\frac{d r_{*}}{d r}=\frac{r^{2}+M^{2} \tilde{a}^{2}}{\Delta} .
$$

and fix the additive constant by choosing

$$
\begin{aligned}
r_{*}=r & +2 M \frac{r_{+}}{r_{+}-r_{-}} \ln \left(\frac{r-r_{+}}{2 M}\right) \\
& -2 M \frac{r_{-}}{r_{+}-r_{-}} \ln \left(\frac{r-r_{-}}{2 M}\right) .
\end{aligned}
$$

$u:=t-r_{*}$ is thus an outgoing null coordinate.

The particle worldline is $x^{i}=x_{\text {particle }}^{i}(t)$; we consider this to be known in advance, i.e., we do not consider changes to the particle worldline induced by the self-force. For present purposes, we consider only particle orbits in the Kerr spacetime's equatorial plane; this restriction is for computational convenience and is not fundamental. We take the particle to orbit in the $d \phi / d t>0$ direction, with $\tilde{a}>0$ for prograde orbits and $\tilde{a}<0$ for retrograde orbits.

We define $r_{\min }$ and $r_{\max }$ to be the particle periapsis and apoapsis $r$ coordinates, respectively. We parametrize bound geodesic equatorial particle orbits by the usual (dimensionless) semilatus rectum $p$ and eccentricity $e$ (defined by $r_{\min }=$ $p M /(1+e)$ and $\left.r_{\max }=p M /(1-e)\right)$, so that the particle orbit is given by

$$
r_{\text {particle }}(t)=\frac{p M}{1+e \cos \chi_{r}(t)},
$$

for a suitable orbital-phase function $\chi_{r}$. We refer to the combination of a spacetime and a (bound geodesic) particle orbit as a "configuration," and parametrize it with the triplet $(\tilde{a}, p, e)$. We define $T_{r}$ to be the coordinate-time period of the particle radial motion; we usually refer to $T_{r}$ as the particle "orbital period."

\section{CALCULATIONS OF SCALAR-FIELD AND GRAVITATIONAL PERTURBATIONS OF KERR SPACETIME}

\section{A. Scalar-field perturbations of Kerr spacetime}

In this section, we summarize TW's scalar-field calculations $[8,34]$. These authors consider a real scalar field $\Phi_{\text {physical }}$ satisfying the wave equation in Kerr spacetime,

$$
\square \Phi_{\text {physical }}=-4 \pi q \int \frac{\delta^{4}\left(x^{a}-x_{\text {particle }}^{a}(t)\right)}{\sqrt{-g}} d \tau,
$$

sourced by a point "particle" of scalar charge $q$ which is taken to move on a (pre-specified) equatorial geodesic orbit. $\Phi_{\text {physical }}$ satisfies outgoing-radiation (retarded) boundary conditions at $\mathcal{J}^{+}$. This system provides a toy model of the full gravitational perturbation problem without the complexity of gauge choice.

Because $\Phi_{\text {physical }}$ diverges on the particle worldline, (5) must be regularized. TW use an effective-source regularization of the type introduced by Barack and Golbourn [43] and Vega and Detweiler [44] (see Ref. [45] for a review), using the puncture function and effective source described by Wardell et al. [34]. In a neighborhood of the particle worldline, TW define a (real) regularized scalar field $\Phi_{\text {regularized }}=\Phi_{\text {physical }}-$ $\Phi_{\text {puncture }}$, where $\Phi_{\text {puncture }}$ is a suitably chosen approximation to the Detweiler-Whiting singular field [46]. The (4-vector) self-force acting on the particle is then given by

$$
F_{a}=\left.\left(\nabla_{a} \Phi_{\text {regularized }}\right)\right|_{x^{i}=x_{\text {particle }}^{i}(t)}{ }^{.}
$$

TW make an azimuthal Fourier decompositions of all the spacetime scalar fields into complex $e^{i m \tilde{\phi}}$ modes,

$$
\Phi(t, r, \theta, \phi)=\sum_{m=-\infty}^{\infty} \frac{1}{r} \varphi_{m}(t, r, \theta) e^{i m \tilde{\phi}},
$$

where the extra factor of $1 / r$ is introduced for computational convenience and where $\tilde{\phi}:=\phi+f(r)$ is an "untwisted" azimuthal coordinate, with

$$
f(r)=\frac{\tilde{a}}{2 \sqrt{1-\tilde{a}^{2}}} \ln \left|\frac{r-r_{+}}{r-r_{-}}\right| .
$$

For each $m$-mode, TW introduce a finite worldtube surrounding the particle worldline in $(t, r, \theta)$ space. For particle orbits with significant eccentricity $(e \gtrsim 0.2)$ the worldtube [now viewed as a region in $(r, \theta)$ in each $t=$ constant slice] moves inward and outward in $r$ during each orbit so as to always contain the particle. All the results reported here were obtained using a worldtube which is rectangular in $(r, \theta)$, with size $10 M$ in $r_{*}$ by approximately 0.25 radians in $\theta$, symmetric about the equatorial plane, and kept centered on the particle to within $0.25 M$ in $r_{*}$ as the particle moves.

TW numerically solve for the piecewise function

$$
\left(\varphi_{m}\right)_{\text {numerical }}= \begin{cases}\left(\varphi_{m}\right)_{\text {regularized }} & \text { inside the worldtube } \\ \left(\varphi_{m}\right)_{\text {physical }} & \text { outside the worldtube }\end{cases}
$$

using a time-domain 2+1-dimensional finite-difference numerical evolution with mesh refinement. Because the (Kerr) background spacetime is axisymmetric, the Fourier modes $\varphi_{m}$ evolve independently - there is no mixing of the modes. Because the physical scalar field $\Phi$ is real, only the $m \geqslant 0$ 
modes need to be explicitly computed; the $m<0$ modes may be obtained by symmetry.

Corresponding to the Fourier decomposition (7), the selfforce (6) can be written as a similar sum of $e^{i m \tilde{\phi}}$ modes,

$$
F_{a}=q \sum_{m=0}^{\infty} F_{a}^{(m)}
$$

where each $F_{a}^{(m)}$ may be computed from the corresponding $\left(\varphi_{m}\right)_{\text {regularized }}$ field near the particle. TW compute a finite set of modes (typically $0 \leqslant m \leqslant 20$ ) and estimate the $m>20$ contributions to the sum (10) via a large- $m$ asymptotic series.

TW use a Zenginoğlu-type hyperboloidal compactification [47-54] so they also have direct access to far-field quantities at $\mathcal{J}^{+}$(where the coordinate $t$ becomes a Bondi-type retarded time coordinate) ${ }^{3}$

\section{B. Gravitational perturbations of Kerr spacetime}

In this section, we summarize the metric reconstruction approach used by van de Meent [35-38] to calculate gravitational perturbation of Kerr spacetime generated by particles on bound geodesic orbits. This approach starts from the spin(-2) Teukolsky variable,

$$
\Phi_{-2}:=(r-i a \cos \theta)^{4} \Psi_{4},
$$

where $\Psi_{4}$ is one of the Weyl scalars. As shown by Teukolsky [41], linear perturbations to this variable satisfy an equation of motion that decouples from all other degrees of freedom. Moreover, unlike the linearized Einstein equation, solutions to the Teukolsky equation can be decomposed into Fourierharmonic modes,

$$
\Phi_{-2}=\sum_{\mathfrak{l} m \omega} R_{\mathfrak{l} m \omega}(r) S_{\mathfrak{l} m \omega}(\theta) e^{i m \phi-i \omega t},
$$

where the $R_{\text {I } m \omega}$ are solutions of the radial Teukolsky equation, the $S_{\mathfrak{l} m \omega}$ are spin-weight spheroidal harmonics, and $\mathfrak{l}$ is the spheroidal mode number. In van de Meent's code, the radial Teukolsky equation can be solved to arbitrarily high precision using a numerical implementation of the semi-analytical methods of Mano, Suzuki, and Takasugi $[55,56]$.

Remarkably, $\Phi_{-2}$ contains almost all information about the corresponding perturbation of the metric [57], and in vacuum it is possible to reconstruct the metric perturbation in a radiation gauge [58-61]. As detailed in Refs. [35-38], this procedure can be used to calculate the backreaction of the metric perturbation on the particle, the gravitational self-force, which then takes the form

$$
F_{a}=\sum_{\substack{\mathfrak{l} m \omega \\ n k \pm}} \mathcal{C}_{a m \omega n k}^{ \pm} R_{\mathfrak{l} m \omega}^{(n) \pm}\left(r_{0}\right) S_{\mathfrak{l} m \omega}^{(k)}\left(\theta_{0}\right) e^{i m \phi_{0}-i \omega t_{0}},
$$

\footnotetext{
${ }^{3}$ More precisely, TW define compactified coordinates $\left(T, R_{*}\right)$ which are identical to (respectively) the Boyer-Lindquist $t$ and the tortoise coordinate $r_{*}$ throughout a neighborhood of the region $r_{\min } \leqslant$ $r \leqslant r_{\max }$ containing the particle orbit, but which are compactified near the event horizon and $\mathcal{J}^{+} . T$ is a Bondi-type retarded time coordinate at $\mathcal{J}^{+}$. For present purposes the details of the compactification are not important, so for convenience of exposition we refer to $T$ as $t$ hereinafter when describing our diagnostics at $\mathcal{J}^{+}$.
}

where the $R_{\mathfrak{l} m \omega}^{ \pm}\left(r_{0}\right)$ are vacuum solutions of the radial Teukolsky equation analytically extended to the particle position $r_{0}$ from either infinity $(+)$ or the black hole horizon $(-)$ (method of extended homogeneous solutions [62]), and the $(n)$ and $(k)$ superscripts on a function denote derivatives with respect to the argument. The indices $n$ and $k$ run from 0 to 3 .

Although each individual term in the sum above is finite, the sum as a whole does not converge. This is a simple consequence of the fact that it was built from the retarded field perturbation rather than the Detweiler-Whiting regular field. To obtain the regular field, we still need to subtract the DetweilerWhiting singular field. In principle, this can be done mode by mode. To match previous analytical calculations of the large- $\ell$ behavior of the singular field $[63,64]$, we need to re-expand the spheroidal $\mathfrak{l}$-modes to spherical $\ell$-modes,

$$
F_{a}^{(\ell)}=\sum_{\substack{\mathfrak{l} m \omega \\ n \pm}} \tilde{\mathcal{C}}_{a \mathfrak{l} m \omega n}^{\ell \pm} R_{\mathfrak{l} m \omega}^{(n) \pm}\left(r_{0}\right) Y_{\ell m}\left(\theta_{0}\right) e^{i m \phi_{0}-i \omega t_{0}} .
$$

With this re-expansion, the local gravitational self force is given by

$$
F_{a}=\sum_{\ell} F_{a}^{(\ell)}-B_{a},
$$

where, as shown in Ref. [65], we can use the Lorenz-gauge $B_{a}$ parameter given in Refs. [63,64].

The metric reconstruction formalism can only recover parts of the metric that contribute to $\Psi_{4}$. This means that the reconstructed metric carries an ambiguity, which can be shown [57] to consist of perturbations of the background within the class of Kerr metrics and pure gauge contributions. These ambiguities can be uniquely fixed based on physical considerations $[66,67]$. The corrections needed to fix these ambiguities are known and straightforward to add. They contribute only to the low frequency envelope of the self-force. Hence, to facilitate identification and extraction of the wiggles in the gravitational self-force, we omit them in this work.

Frequency domain calculations of the type used here are ideally suited for calculating metric perturbations with a sparse discrete frequency spectrum, such as those sourced by a particle on a low eccentricity geodesic. That spectrum becomes denser at higher eccentricities, necessitating the calculation of more frequency modes and making the calculation more time-consuming. Moreover, as discussed in detail in Ref. [36], the method of extended homogeneous solutions leads to large cancellations between different (low) frequency modes for high- $\ell$ modes, causing a large loss of precision. In this work, we tackle this problem by harnessing the full power of the arbitrary precision implementation of our code and simply throw more precision at the computation than we lose in the cancellation.

For this work we calculated the full gravitational self-force for orbits with eccentricities up to $e=0.8$, which involves dealing with cancellations of around 30 orders of magnitude. These calculations are fairly resource intensive, taking $\mathcal{O}\left(10^{4}\right)$ CPU hours (or a few days on $400 \mathrm{CPUs}$ ) to compute.

However, for many aspects of our investigation here, we do not need the full local regular metric perturbation. If we want to look for the dominant low-l QNMs, then these will 
TABLE I. This table shows the local- and radiated-field diagnostics in which we study wiggles.

\begin{tabular}{llc}
\hline \hline Field perturbation at $\ldots$ & Scalar field & Gravitational \\
\hline fixed spatial position & $\left(\varphi_{m}\right)_{\text {regularized }}$ & $\Phi_{-2}^{(\mathrm{I} m)}$ \\
(strong-field) & & \\
particle position & $F_{a}^{(m)}$ & ${ }_{-2} F_{a}^{(\mathrm{I} m)}$ \\
$\mathcal{J}^{+}$ & $\partial_{t t}\left(\left(\varphi_{m}\right)_{\text {physical }}\right)$ & $\Psi_{4}$ \\
\hline \hline
\end{tabular}

contribute (mostly) to the low-l modes of the gravitational metric perturbation. For this purpose, we define the individual l $m$ modes of the Teukolsky variable

$$
\Phi_{-2}^{(\mathfrak{l} m)}=\sum_{\omega} R_{\mathfrak{l} m \omega}(r) S_{\mathfrak{l} m \omega}(\theta) e^{i m \phi-i \omega t},
$$

and the gravitational self-force

$$
F_{a}^{(\mathrm{l} m)}:=\sum_{\substack{\omega \\ n k \pm}} \mathcal{C}_{a m \omega n k}^{ \pm} R_{\mathfrak{l} m \omega}^{(n) \pm}\left(r_{0}\right) S_{\mathfrak{l} m \omega}^{(k)}\left(\theta_{0}\right) e^{i m \phi_{0}-i \omega t_{0}} .
$$

These are much easier to compute, and for low $\mathfrak{l}$ do not suffer from the large loss of precision due to the method of extended homogeneous solutions, thus allowing for very high accuracy calculations without excessive computational cost.

\section{FIELD DIAGNOSTICS}

We consider several different types of local- and radiatedfield diagnostics, and attempt to fit the wiggles in these diagnostics to QNM models. Clearly the presence of wiggles in the physical scalar field or metric perturbation implies the presence of wiggles in some or all of the $e^{i m \tilde{\phi}}$ scalarfield modes or $(\mathfrak{l} m)$ metric-perturbation modes (respectively) and vice versa. ${ }^{4}$ Because many fewer QNMs are present at significant amplitude (usually only one, or in a few cases two), it is much simpler to analyze wiggles in the individual modes.

Table I summarizes our local- and radiated-field diagnostics for studying wiggles. We consider (time series of) diagnostics at three locations in spacetime:

(1) Diagnostics of the local field at selected fixed spatial "watchpoint" coordinate positions $(r, \theta, \phi)=$ constant. These diagnostics directly sample any QNMs that may be present, but the diagnostics may be contaminated by the direct field when the particle is close to the watchpoint position.

For the scalar-field case, we avoid any such possible contamination by considering the regularized field mode $\left(\varphi_{m}\right)_{\text {regularized }}$. However, this is only defined within the worldtube, so for orbits with significant eccentricity (where the worldtube moves in $(r, \theta)$ during the particle orbit) any given watchpoint may lie outside the worldtube (and thus leave $\left(\varphi_{m}\right)_{\text {regularized }}$ undefined) during some parts of the orbit. To minimize this effect, for many of the analyses reported here

\footnotetext{
${ }^{4}$ While it would be theoretically possible for multiple modes to have wiggles of the same frequency whose amplitudes sum to approximately zero (leading to an absence of wiggles in the physical fields), in practice, we have never observed this.
}

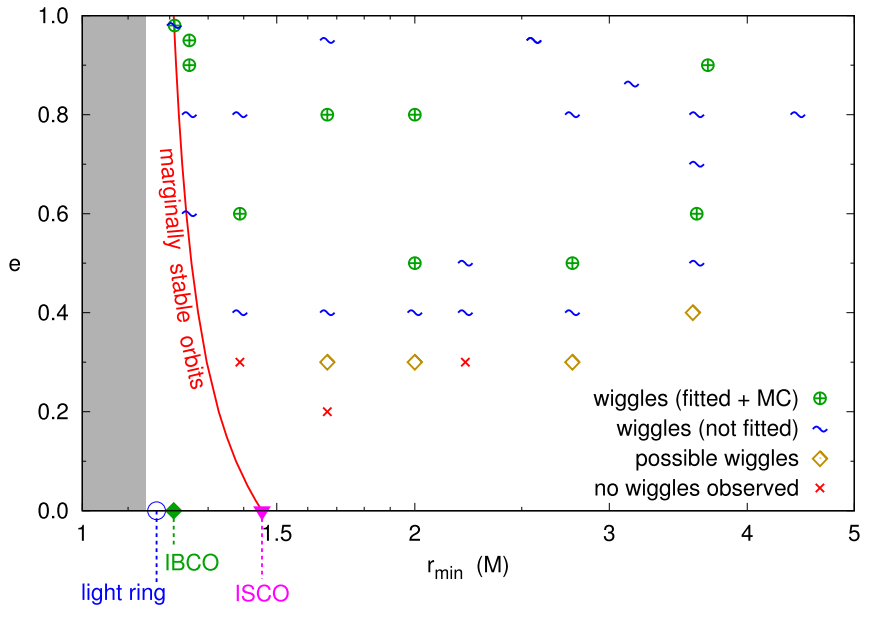

FIG. 1. This figure shows the phase space of the Kerr spin $\tilde{a}=$ 0.99 scalar-field configurations presented here, plotted in terms of the periapsis radius $r_{\min }$ and the eccentricity $e$. The shaded region at the left shows orbits with periapsis inside the horizon. The light ring, innermost bound circular orbit (IBCO), innermost stable circular orbit (ISCO), and the locus of marginally stable orbits are also shown. The meanings of the plot symbols are described in detail in Table III.

we use watchpoint positions which are near the orbit apoapsis, where the particle (and hence the worldtube) motion is relatively slow and hence a suitable watchpoint can remain within the worldtube for a relatively long time in each orbit. All our scalar-field watchpoints are in the equatorial plane.

For the gravitational case, the regularized field is not readily available, so instead we have the code output the retarded $\Phi_{-2}^{(\mathrm{l} m)}$ on the symmetry axis of the background Kerr spacetime $(\theta=0)$ and the equatorial plane $(\theta=\pi / 2)$ at coordinate radii corresponding to the particle periapsis and apoapsis distances.

(2) Diagnostics of the local field at the particle. Here we consider the $e^{i m \tilde{\phi}}$ (scalar-field) and (l $m$ ) (gravitational) modes of the self-force itself. The main complication here is that these diagnostics sample the field perturbation at a timedependent position (the particle position), so our fitting model for the QNM effects must include corrections for the spatial variation of the QNM eigenfunctions as the particle (sampling point) moves. For the azimuthal $(\phi)$ particle motion, this is straightforward (described in Sec. IV) but for the radial $(r)$ motion we include this correction only approximately.

(3) Diagnostics of the radiated field at $\mathcal{J}^{+}$. These have the advantage of being physically observable and of allowing the $e^{i m \tilde{\phi}}$ (scalar-field) and $(\mathfrak{l} \mathrm{m})$ (gravitational) mode decompositions to be defined in a weak-field region (for the gravitational case, this also avoids any gauge dependence).

At $\mathcal{J}^{+}$, we only have the physical (retarded) fields available, so it is more difficult to separate wiggles from the overall radiation pattern. To help in making this separation, we observe that wiggles are of relatively high (temporal) frequency relative to other major features in the radiated fields, so that taking time derivatives of the radiated fields increases the wiggles' amplitude relative to that of the other features. We have found that good results are obtained by using as diagnostics the second time derivatives $\partial_{t t}\left(\left(\varphi_{m}\right)_{\text {physical }}\right)$ evaluated in the 

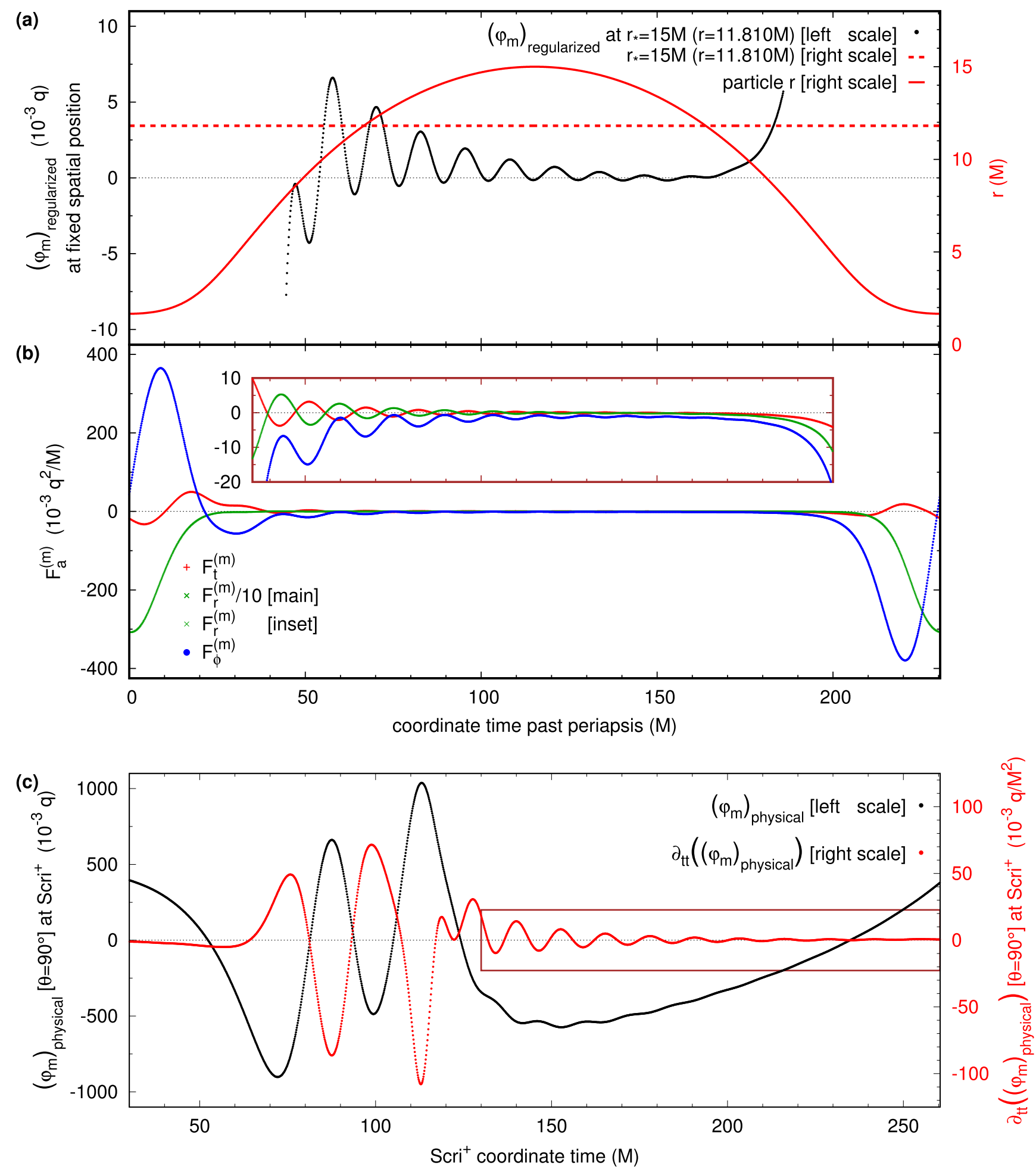

FIG. 2. This figure shows wiggles in the $m=1$ scalar-field diagnostics for a single particle orbit $\left(t \in\left[38 T_{r}, 39 T_{r}\right]\right)$ of the $(\tilde{a}, p, e)=$ $(0.99,3,0.8)$ configuration. In (b) and (c), the inset boxes (which share common timescales with their corresponding main figures) show the regions where wiggles are visible. (a) shows the regularized scalar field at $r_{*}=15 M(r=11.810 M)$, at times when this position is within the worldtube. (b) shows the three nontrivial coordinate components of the 4-vector self-force $F_{a}^{(m)}$. (c) shows the physical scalar field and its second time derivative, on the equator at $\mathcal{J}^{+}$; the fields at other viewing angles are very similar in shape but with smaller amplitudes. Due to the orbital precession, the $\mathcal{J}^{+}$fields are not "periodic with period $T_{r}$." Note that the zero point of the $\mathcal{J}^{+}$time coordinate does not correspond to periapsis. 

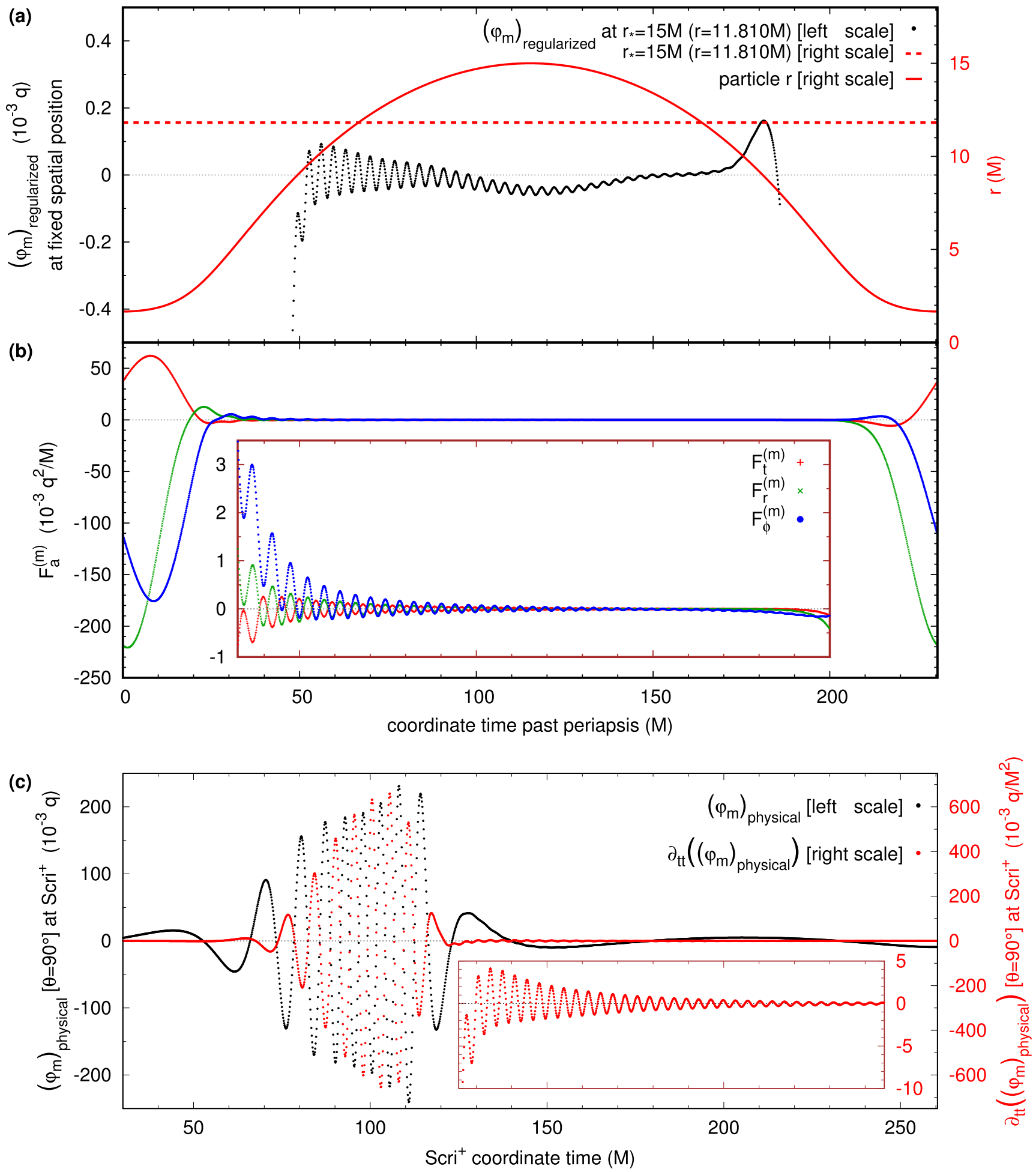

FIG. 3. This figure shows wiggles in the $m=4$ scalar-field diagnostics for a single particle orbit $\left(t \in\left[13 T_{r}, 14 T_{r}\right]\right)$ of the $(\tilde{a}, p, e)=$ $(0.99,3,0.8)$ configuration. In (b) and (c), the inset boxes (which share common timescales with their corresponding main figures) show the regions where wiggles are visible. (a) shows the regularized scalar field at $r_{*}=15 M(r=11.810 M)$, at times when this position is within the worldtube. (b) shows the three nontrivial coordinate components of the 4-vector self-force $F_{a}^{(m)}$. (c) shows the physical scalar field and its second time derivative, on the equator at $\mathcal{J}^{+}$; the fields at other viewing angles are very similar in shape but with smaller amplitudes. Due to the orbital precession, the $\mathcal{J}^{+}$fields are not "periodic with period $T_{r}$." Note that the zero point of the $\mathcal{J}^{+}$time coordinate does not correspond to periapsis. 


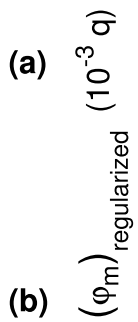

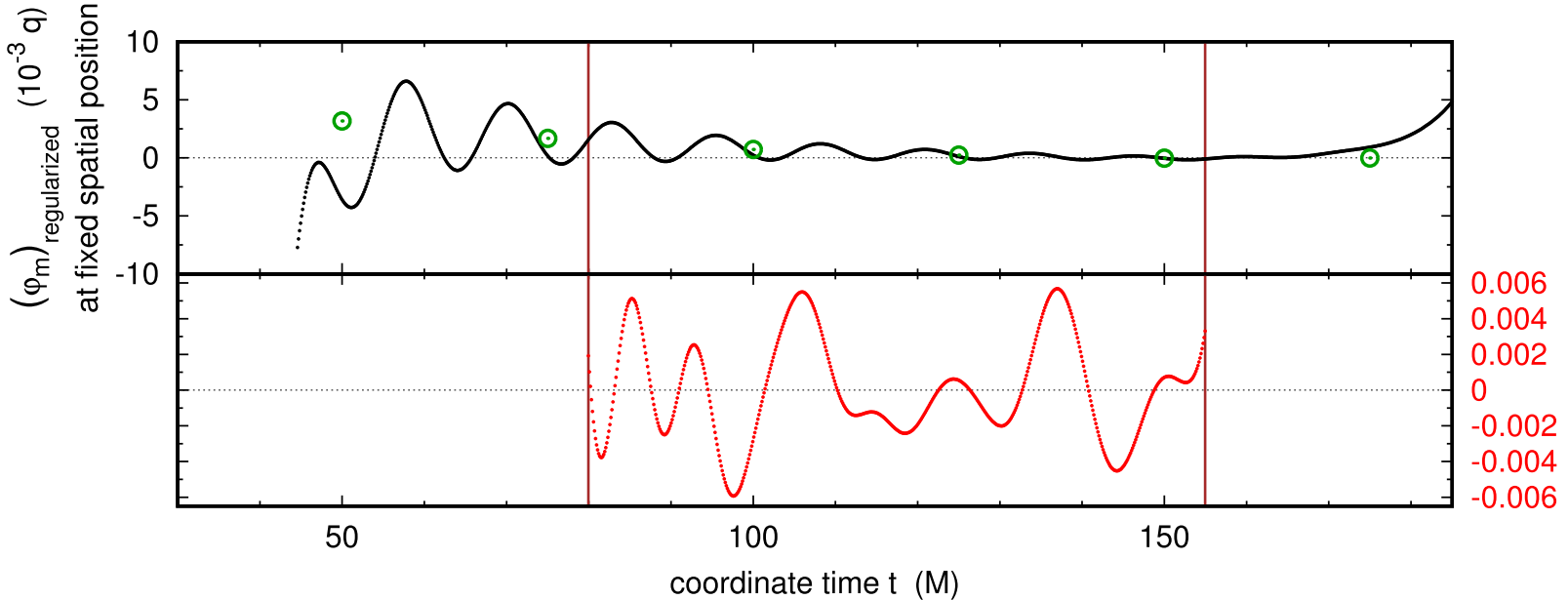

(c)

(d)
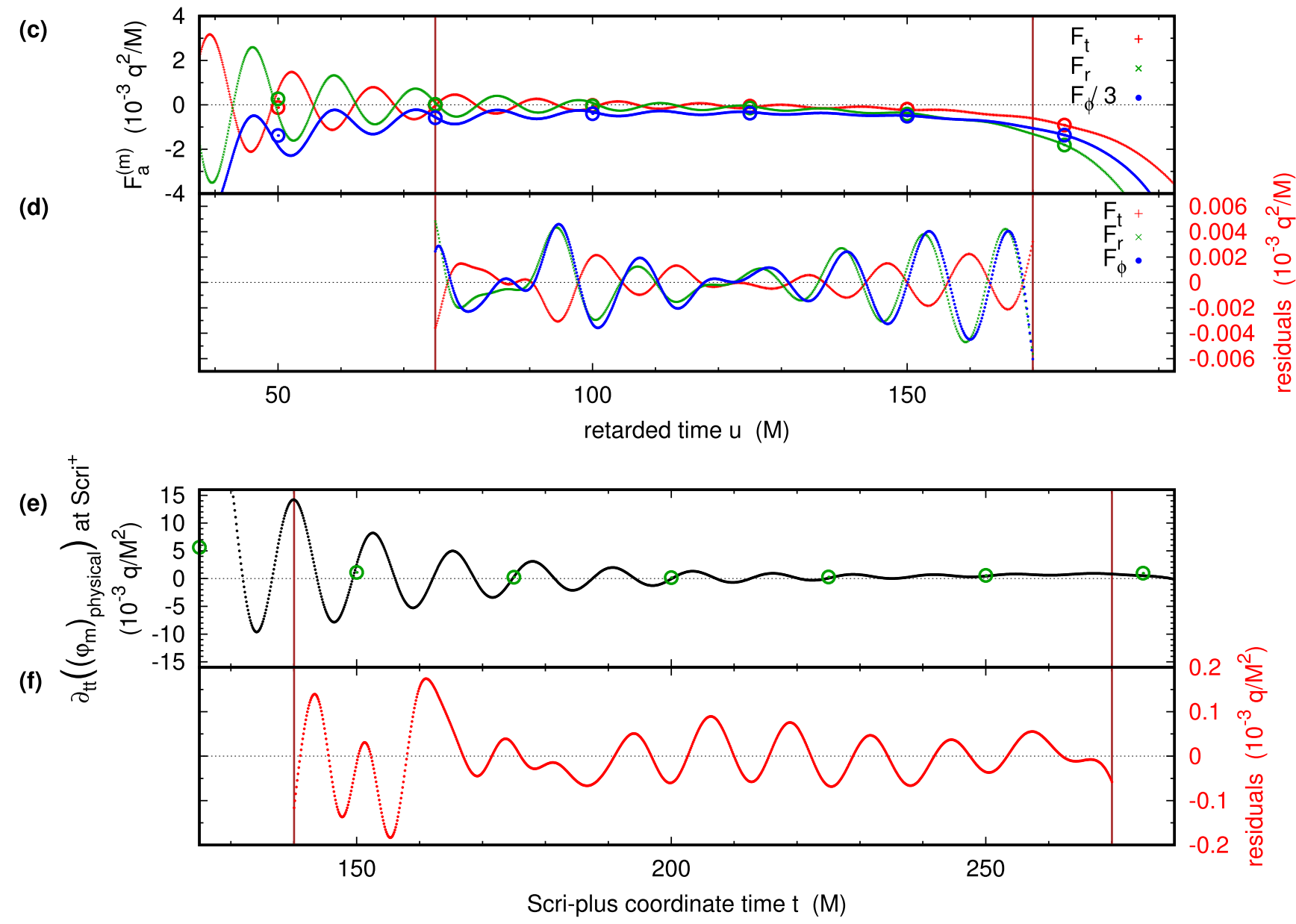

FIG. 4. This figure shows the wiggles and fit residuals for the $m=1$ scalar-field diagnostics for a single particle orbit $\left(t \in\left[38 T_{r}, 39 T_{r}\right]\right)$ of the $(\tilde{a}, p, e)=(0.99,3,0.8)$ configuration. (a) shows the regularized scalar field at $r_{*}=15 M(r=11.810 M)$ as a function of coordinate time $t$, at times when this position is within the worldtube, while (b) shows the residuals after fitting this data to the model (18). (c) shows the three nontrivial coordinate components of the 4-vector self-force $F_{a}^{(m)}$ as a function of retarded time $u$, while (d) shows the residuals after fitting this data to the model (19). (e) shows the second time derivative of the physical scalar field on the equator at $\mathcal{J}^{+}$as a function of the $\mathcal{J}^{+}$coordinate time $t$, while (f) shows the residuals after fitting these data to the model (20). In each subfigure, the vertical tan lines mark the interval over which the model is fitted. In (a), (c), and (e), the circles show show the background spline control points; the differences between the data and the fitted models are too small to be visible at the scales of these subplots. 
(a)

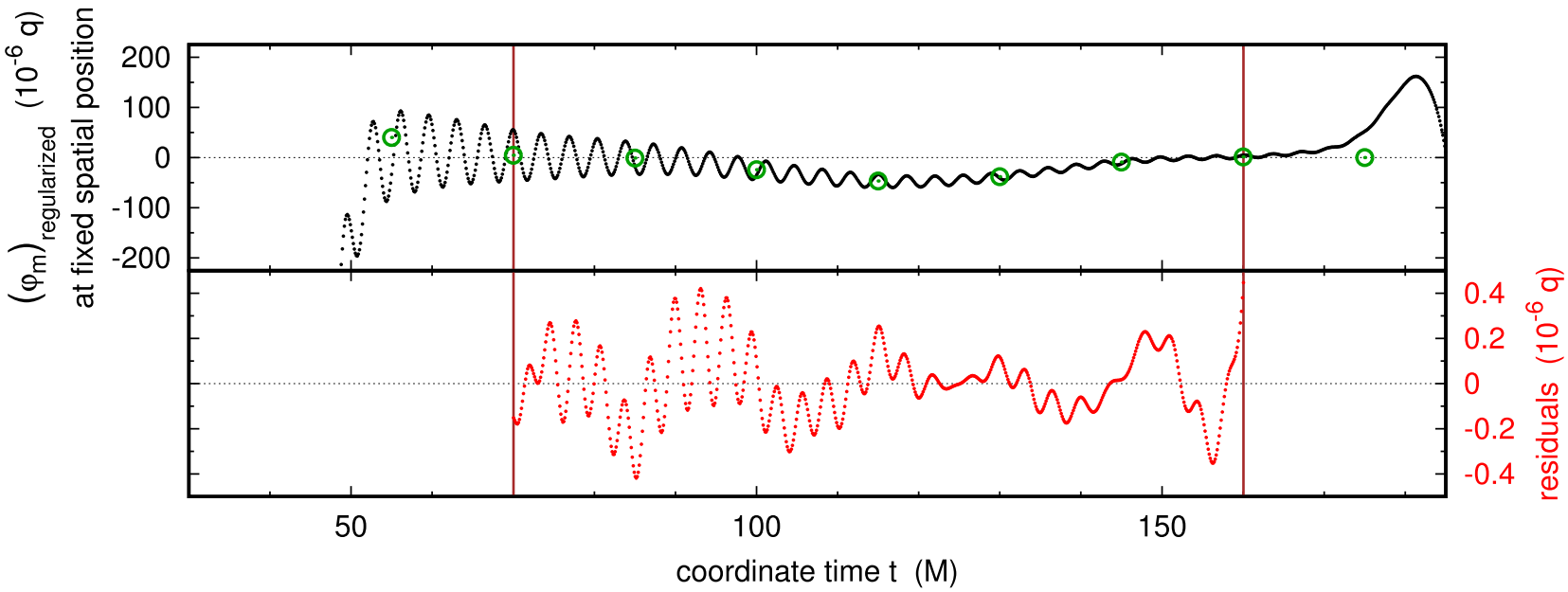

(c)
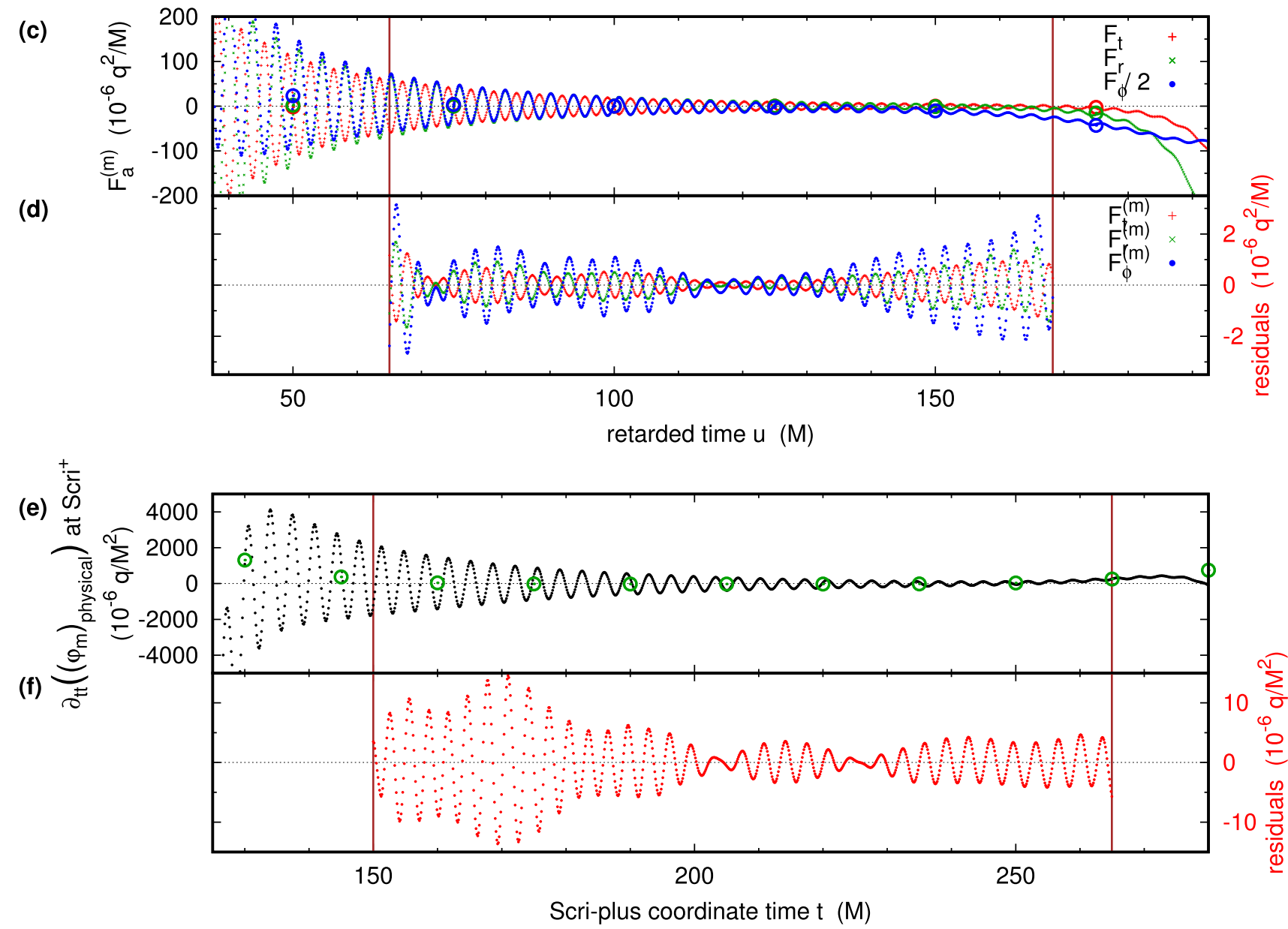

FIG. 5. This figure shows the wiggles and fit residuals for the $m=4$ scalar-field diagnostics for a single particle orbit $\left(t \in\left[13 T_{r}, 14 T_{r}\right]\right)$ of the $(\tilde{a}, p, e)=(0.99,3,0.8)$ configuration. (a) shows the regularized scalar field at $r_{*}=15 M(r=11.810 M)$ as a function of coordinate time $t$, at times when this position is within the worldtube, while (b) shows the residuals after fitting to the model (18). (c) shows the three nontrivial coordinate components of the 4-vector self-force $F_{a}^{(m)}$ as a function of retarded time $u$, while (d) shows the residuals after fitting to the model (19). (e) shows the second time derivative of the physical scalar field on the equator at $\mathcal{J}^{+}$as a function of the $\mathcal{J}^{+}$coordinate time $t$, while (f) shows the residuals after fitting to the model (20). In each subfigure, the vertical tan lines mark the interval over which the model is fitted. In (a), (c), and (e), the circles show show the background spline control points; the differences between the data and the fitted models are too small to be visible at the scales of these subplots. 
TABLE II. This table describes the Kerr scalar-field configurations surveyed here. The table rows are ordered by decreasing Kerr spin $\tilde{a}$ (horizontal lines separate groups of rows for the same spin), within this by increasing particle periapsis radius $r_{\text {min }}$, and within this by decreasing particle orbital eccentricity $e$. $(\tilde{a}, p, e)$ describe the configuration. $T_{r}$ is the coordinate-time period of the orbit radial motion, $r_{\text {min }}$ is the particle periapsis $r$ coordinate, and $\dot{\phi}_{\text {periapsis }}$ is the particle angular 3-velocity in $\phi$ (i.e., $\left.d \phi / d t\right)$ at the point of periapsis. $r_{\text {watchpoint }}$ is the $r$ coordinate of the watchpoint used for observing wiggles, or blank if no watchpoint data was available for this configuration. "Resolution" is the highest numerical resolution used for simulations of this configuration, and refers to the grid structures described in Table IV. The final sets of columns describe the presence or absence of wiggles in our field diagnostics for $m=1$ through $m=6$; for each $m$, there are 3 columns labeled "w," " $F$," and " $\mathcal{J}$," describing (respectively) wiggles in the regularized field $\left(\varphi_{m}\right)_{\text {regularized }}$ at a fixed "watchpoint" (located on the equator at $\left.r=r_{\text {watchpoint }}\right)$, wiggles in the self-force $F_{a}$, and wiggles in the second time derivative of the physical field, $\partial_{t t}\left(\left(\varphi_{m}\right)_{\text {physical }}\right)$, at $\mathcal{J}^{+}$. The meanings of the symbols in these columns are described in detail in Table III; briefly, $\oplus$ or + means that we observed wiggles and fit them to the appropriate model described in Sec. IV A, $\sim$ means that we observed wiggle-like oscillations but did not attempt a quantitative fit, $\diamond$ means that we observed oscillations which might be wiggles but were not clearly separated from the background variation, and $\mathrm{x}$ means that we did not observe wiggles (n.b. this does not prove the absence of wiggles, only that we did not observe them).

\begin{tabular}{|c|c|c|c|c|c|c|c|c|c|c|c|c|c|c|c|c|c|c|c|c|c|c|c|c|c|c|}
\hline \multirow[b]{2}{*}{ Name } & \multirow[b]{2}{*}{$\tilde{a}$} & \multirow[b]{2}{*}{$p$} & \multirow[b]{2}{*}{$e$} & \multirow{2}{*}{$\begin{array}{c}T_{r} \\
(M)\end{array}$} & \multirow{2}{*}{$\begin{array}{l}r_{\min } \\
(M)\end{array}$} & \multirow{2}{*}{$\begin{array}{c}\dot{\phi}_{\text {periapsis }} \\
\left(M^{-1}\right)\end{array}$} & \multirow{2}{*}{$\begin{array}{c}r_{\text {watchpoint }} \\
(M)\end{array}$} & \multirow[b]{2}{*}{ Resolution } & \multicolumn{3}{|c|}{$\mathrm{m}=1$} & \multicolumn{3}{|c|}{$\mathrm{m}=2$} & \multicolumn{3}{|c|}{$\mathrm{m}=3$} & \multicolumn{3}{|c|}{$\mathrm{m}=4$} & \multicolumn{3}{|c|}{$m=5$} & & $\mathrm{~m}=$ & \\
\hline & & & & & & & & & $\mathrm{w}$ & $F$ & $\mathcal{J}$ & w & $F$ & $\mathcal{J}$ & $\mathrm{w}$ & $F$ & $\mathcal{J}$ & $\mathrm{w}$ & $F$ & $\mathcal{J}$ & $\mathrm{w}$ & $F$ & $\mathcal{J}$ & $\mathrm{w}$ & $F$ & $\mathcal{J}$ \\
\hline w9x5-161 & 0.99999 & 2.918315 & 0.807519 & 230.442 & 1.615 & 0.344414 & 11.810 & dro12-96 & $\sim$ & $\sim$ & & $\sim$ & $\sim$ & & $\sim$ & $\sim$ & & $\sim$ & $\sim$ & & $\sim$ & $\sim$ & & $\sim$ & $\sim$ & \\
\hline w9x4-368 & 0.9999 & 7.0 & 0.9 & 1513.112 & 3.684 & 0.145444 & 46.304 & dro8-64 & $\oplus$ & $\oplus$ & $\oplus$ & $\oplus$ & $\oplus$ & $\oplus$ & & $\oplus$ & $\oplus$ & & $\oplus$ & $x$ & & $x$ & $x$ & & $x$ & $\mathbf{x}$ \\
\hline w999-278 & 0.999 & 5.0 & 0.8 & 400.508 & 2.778 & 0.199135 & 21.448 & dro10-80 & $\oplus$ & $\oplus$ & $\oplus$ & $\oplus$ & $\oplus$ & $\oplus$ & $\oplus$ & $\oplus$ & $\oplus$ & & & & & & & & & \\
\hline w999-368 & 0.999 & 7.0 & 0.9 & 1513.179 & 3.684 & 0.145443 & & dro8-64 & & $\oplus$ & $\oplus$ & & $\oplus$ & $\oplus$ & & $\oplus$ & & & $\sim$ & $x$ & & $x$ & $x$ & & & $x$ \\
\hline ze98a & 0.99 & 2.3981 & 0.98 & 3414.259 & 1.211 & 0.430498 & 61.223 & dro6-48 & $\sim$ & $\sim$ & & $\sim$ & $\sim$ & & $\sim$ & $\sim$ & & $\sim$ & $\sim$ & & $\sim$ & $\sim$ & & $\sim$ & $\sim$ & \\
\hline ze98 & 0.99 & 2.4 & 0.98 & 3304.620 & 1.212 & 0.430300 & & dro10-80 & & $\oplus$ & $\oplus$ & & $\oplus$ & $\oplus$ & & $\oplus$ & $\oplus$ & & $\oplus$ & $\oplus$ & & $\oplus$ & $\oplus$ & & $\oplus$ & $\sim$ \\
\hline w99-125a & 0.99 & 2.4375 & 0.95 & 957.757 & 1.25 & 0.421924 & 36.314 & dro8-64 & $\oplus$ & $\oplus$ & $\oplus$ & $\oplus$ & $\oplus$ & $\oplus$ & $\oplus$ & $\oplus$ & $\oplus$ & & $\sim$ & $\sim$ & & & & & & \\
\hline w99-125b & 0.99 & 2.375 & 0.9 & 432.084 & 1.25 & 0.421555 & 20.543 & dro8-64 & $\oplus$ & $\oplus$ & $\oplus$ & $\oplus$ & $\oplus$ & $\oplus$ & $\oplus$ & $\oplus$ & $\oplus$ & $\oplus$ & $\oplus$ & $\oplus$ & & $\sim$ & & & & \\
\hline w99-125c & 0.99 & 2.25 & 0.8 & 247.845 & 1.25 & 0.420811 & 8.595 & dro6-48 & $\sim$ & $\sim$ & & $\sim$ & $\sim$ & & $\sim$ & $\sim$ & & $\sim$ & $\sim$ & & $\sim$ & $\sim$ & & $\sim$ & $\sim$ & \\
\hline w99-125d & 0.99 & 2.0 & 0.6 & 228.354 & 1.25 & 0.419300 & 3.003 & dro8-64 & $\sim$ & $\sim$ & & $\sim$ & $\sim$ & & $\sim$ & $\sim$ & & $\sim$ & $\sim$ & & $\sim$ & $\sim$ & & & & \\
\hline w99-139 & 0.99 & 2.5 & 0.8 & 211.605 & 1.389 & 0.389231 & & dro8-64 & & $\sim$ & & & $\sim$ & & & $\sim$ & & & $\sim$ & & & $\sim$ & & & $\sim$ & \\
\hline w99-139b & 0.99 & 2.222222 & 0.6 & 133.939 & 1.389 & 0.386760 & 3.926 & dro8-64 & + & + & + & + & $\oplus$ & $\oplus$ & $\oplus$ & $\oplus$ & $\oplus$ & $\oplus$ & $\oplus$ & $\oplus$ & $\sim$ & & $\sim$ & & & \\
\hline w99-139d & 0.99 & 1.944444 & 0.4 & 126.071 & 1.389 & 0.384220 & 1.984 & dro6-48 & $x$ & $\diamond$ & & $x$ & $\sim$ & & $x$ & $\sim$ & & $x$ & & & $x$ & & & $x$ & & \\
\hline w99-139c & 0.99 & 1.805556 & 0.3 & 135.092 & 1.389 & 0.382960 & 1.743 & dro6-48 & $x$ & $x$ & & $x$ & $x$ & & $x$ & $x$ & & $x$ & $x$ & & $x$ & $x$ & & $x$ & $x$ & \\
\hline w99-167m & 0.99 & 3.25 & 0.95 & 1329.680 & 1.667 & 0.336304 & 47.740 & dro8-64 & $\sim$ & $\sim$ & & $\sim$ & $\sim$ & & $\sim$ & $\sim$ & & $\sim$ & $\sim$ & & $\sim$ & $\sim$ & & & & \\
\hline w99-167 & 0.99 & 3.0 & 0.8 & 230.442 & 1.667 & 0.333682 & 11.810 & dro10-80 & $\oplus$ & $\oplus$ & $\oplus$ & $\oplus$ & $\oplus$ & $\oplus$ & $\oplus$ & $\oplus$ & $\oplus$ & $\oplus$ & $\oplus$ & $\oplus$ & $\oplus$ & $\oplus$ & $\oplus$ & $\oplus$ & $\oplus$ & $\oplus$ \\
\hline w99-167k & 0.99 & 2.333333 & 0.4 & 100.014 & 1.667 & 0.326143 & 2.332 & dro6-48 & $\diamond$ & $\diamond$ & & $x$ & $\sim$ & & $x$ & $\sim$ & & $x$ & $\diamond$ & & $x$ & $x$ & & $x$ & & \\
\hline w99-167d & 0.99 & 2.166667 & 0.3 & 97.092 & 1.667 & 0.324141 & 2.332 & dro6-48 & $x$ & $\diamond$ & & $x$ & $\diamond$ & & $x$ & $\diamond$ & & $x$ & $x$ & & $x$ & $x$ & & $x$ & $x$ & \\
\hline w99-167j & 0.99 & 2 & 0.2 & 98.818 & 1.667 & 0.322115 & 1.984 & dro6-48 & $x$ & $x$ & & $x$ & $x$ & & $x$ & $x$ & & $x$ & $x$ & & $x$ & $x$ & & $x$ & $x$ & \\
\hline w99-200d & 0.99 & 3.6 & 0.8 & 273.551 & 2.0 & 0.281145 & 14.784 & dro8-64 & $\oplus$ & $\oplus$ & $\oplus$ & $\oplus$ & $\oplus$ & $\oplus$ & $\oplus$ & $\oplus$ & $\oplus$ & $\oplus$ & $\oplus$ & $\oplus$ & $\oplus$ & & & & & \\
\hline w99-200 & 0.99 & 3.0 & 0.5 & 111.575 & 2.000 & 0.274441 & & dro6-48 & & $\oplus$ & & & $\oplus$ & & & $\oplus$ & & & $\oplus$ & & & $x$ & & & $x$ & \\
\hline w99-200b & 0.99 & 2.8 & 0.4 & 99.000 & 2.000 & 0.272062 & 3.003 & dro6-48 & $x$ & $\sim$ & & $x$ & $\sim$ & & $x$ & $\sim$ & & $x$ & $\diamond$ & & $x$ & $x$ & & $x$ & $x$ & \\
\hline w99-200c & 0.99 & 2.6 & 0.3 & 91.817 & 2.000 & 0.269607 & 2.636 & dro6-48 & $x$ & $\diamond$ & & $x$ & $x$ & & $x$ & $x$ & & $x$ & $x$ & & $x$ & $x$ & & $x$ & $x$ & \\
\hline w99-222b & 0.99 & 3.333333 & 0.5 & 117.908 & 2.222 & 0.245832 & 4.193 & dro6-48 & $\sim$ & $\sim$ & & $\sim$ & $\sim$ & & $\diamond$ & $\sim$ & & $x$ & & & $x$ & & & $x$ & & \\
\hline w99-222 & 0.99 & 3.111111 & 0.4 & 102.677 & 2.222 & 0.243319 & 3.926 & dro6-48 & $\sim$ & $\sim$ & & $x$ & $\sim$ & & $x$ & $\sim$ & & $x$ & $x$ & & $x$ & $x$ & & $x$ & $x$ & \\
\hline w99-222c & 0.99 & 2.888889 & 0.3 & 93.395 & 2.222 & 0.240719 & 3.003 & dro6-48 & $x$ & $x$ & & $x$ & $x$ & & $x$ & $x$ & & $x$ & $x$ & & $x$ & $x$ & & $x$ & $x$ & \\
\hline e95 & 0.99 & 5.0 & 0.95 & 2436.050 & 2.564 & 0.220775 & & dro8-64 & & $\sim$ & & & $\sim$ & & & $\sim$ & & & $\sim$ & & & $\sim$ & & & & \\
\hline w99-278 & 0.99 & 5.0 & 0.8 & 401.302 & 2.778 & 0.199076 & 20.543 & dro8-64 & $\sim$ & $\sim$ & & $\sim$ & $\sim$ & & $\sim$ & $\sim$ & & $\sim$ & $\sim$ & & $x$ & $\sim$ & & & & \\
\hline w99-278b & 0.99 & 4.166667 & 0.5 & 139.595 & 2.778 & 0.191854 & 5.712 & dro8-64 & $\oplus$ & $\oplus$ & $\oplus$ & $\diamond$ & $\oplus$ & $\oplus$ & $x$ & $\oplus$ & $x$ & $x$ & $x$ & $x$ & $x$ & $x$ & $x$ & $x$ & $x$ & $x$ \\
\hline w99-278c & 0.99 & 3.888889 & 0.4 & 117.842 & 2.778 & 0.189278 & 4.193 & dro6-48 & $\diamond$ & $\sim$ & & $x$ & $\sim$ & & $x$ & $x$ & & $x$ & $x$ & & $x$ & $x$ & & $x$ & $x$ & \\
\hline w99-278d & 0.99 & 3.611111 & 0.3 & 103.835 & 2.778 & 0.186607 & 3.672 & dro6-48 & $x$ & $\diamond$ & & $x$ & $x$ & & $x$ & $x$ & & $x$ & $x$ & & $x$ & $x$ & & $x$ & $x$ & \\
\hline s99 & 0.99 & 5.850762 & 0.861866 & 771.968 & 3.142 & 0.174409 & & dro6-48 & & $\sim$ & & & $\sim$ & & & $\sim$ & & & $\sim$ & & & & & & & \\
\hline w99-357 & 0.99 & 5.0 & 0.4 & 146.751 & 3.571 & 0.139748 & & dro6-48 & & $\diamond$ & & & $x$ & & & $x$ & & & $x$ & & & $x$ & & & $x$ & \\
\hline w99-360c & 0.99 & 6.48 & 0.8 & 560.918 & 3.6 & 0.147411 & & dro6-48 & & $\sim$ & & & $\sim$ & & & $\sim$ & & & $x$ & & & & & & & \\
\hline w99-360b & 0.99 & 6.12 & 0.7 & 330.647 & 3.6 & 0.145260 & & dro6-48 & & $\sim$ & & & $\sim$ & & & $\sim$ & & & $x$ & & & $x$ & & & $x$ & \\
\hline w99-360a & 0.99 & 5.76 & 0.6 & 232.413 & 3.6 & 0.143040 & 11.810 & dro8-64 & $\oplus$ & $\oplus$ & $\oplus$ & $x$ & $\oplus$ & $\oplus$ & $x$ & $x$ & $x$ & $x$ & $x$ & $x$ & $x$ & $x$ & $x$ & $x$ & $x$ & $x$ \\
\hline w99-360j & 0.99 & 5.4 & 0.5 & 179.842 & 3.6 & 0.140745 & 7.835 & dro6-48 & $\diamond$ & $\sim$ & & $x$ & $x$ & $x$ & $x$ & $x$ & $x$ & $x$ & & $x$ & & & & & & \\
\hline e9 & 0.99 & 7.0 & 0.9 & 1513.855 & 3.684 & 0.145429 & 25.105 & dro8-64 & $\oplus$ & $\oplus$ & $\oplus$ & $\oplus$ & $\oplus$ & $\oplus$ & $x$ & $\oplus$ & $\oplus$ & $x$ & $\oplus$ & $x$ & $x$ & $x$ & $x$ & $x$ & $x$ & $x$ \\
\hline w99-444 & 0.99 & 8.0 & 0.8 & 745.170 & 4.444 & 0.113763 & & dro6-48 & & $\sim$ & & & $x$ & & & $x$ & & & $x$ & & & $x$ & & & $x$ & \\
\hline w95-368 & 0.95 & 7.0 & 0.9 & 1516.962 & 3.684 & 0.145349 & & dro6-48 & & $\oplus$ & & & $\oplus$ & & & $\oplus$ & & & $\oplus$ & & & $x$ & & & $x$ & \\
\hline n96 & 0.9 & 5.5 & 0.6 & 227.038 & 3.4375 & 0.151199 & & dro8-64 & & $\sim$ & & & $\sim$ & & & $x$ & & & $x$ & & & $x$ & & & $x$ & \\
\hline w9-368 & 0.9 & 7.0 & 0.9 & 1521.097 & 3.684 & 0.145202 & & dro6-48 & & $\oplus$ & $\oplus$ & & $\oplus$ & $\oplus$ & & $\oplus$ & $\oplus$ & & $\oplus$ & $\oplus$ & & $x$ & $x$ & & $x$ & $x$ \\
\hline
\end{tabular}


TABLE II. (Continued.)

\begin{tabular}{|c|c|c|c|c|c|c|c|c|c|c|c|c|c|c|c|c|c|c|c|c|c|c|c|c|c|c|}
\hline \multirow[b]{2}{*}{ Name } & \multirow[b]{2}{*}{$\tilde{a}$} & \multirow[b]{2}{*}{$p$} & \multirow[b]{2}{*}{$e$} & \multirow{2}{*}{$\begin{array}{c}T_{r} \\
(M)\end{array}$} & \multirow{2}{*}{$\begin{array}{l}r_{\min } \\
(M)\end{array}$} & \multirow{2}{*}{$\begin{array}{c}\dot{\phi}_{\text {periapsis }} \\
\left(M^{-1}\right)\end{array}$} & \multirow{2}{*}{$\begin{array}{c}r_{\text {watchpoint }} \\
(M)\end{array}$} & \multirow[b]{2}{*}{ Resolution } & \multicolumn{3}{|c|}{$\mathrm{m}=1$} & \multicolumn{3}{|c|}{$\mathrm{m}=2$} & \multicolumn{3}{|c|}{$\mathrm{m}=3$} & \multicolumn{3}{|c|}{$\mathrm{m}=4$} & \multicolumn{3}{|c|}{$\mathrm{m}=5$} & \multicolumn{3}{|c|}{$\mathrm{m}=6$} \\
\hline & & & & & & & & & w & $F$ & $\mathcal{J}$ & $\mathrm{w}$ & $F$ & $\mathcal{J}$ & $\mathrm{w}$ & $F$ & $\mathcal{J}$ & $\mathrm{w}$ & $F$ & $\mathcal{J}$ & $\mathrm{w}$ & $F$ & $\mathcal{J}$ & w & $F$ & $\mathcal{J}$ \\
\hline n95 & 0.9 & 10.0 & 0.5 & 378.408 & 6.667 & 0.062994 & & dro8-64 & & $x$ & & & $x$ & & & $x$ & & & $x$ & & & $x$ & & & $x$ & \\
\hline w8-368 & 0.8 & 7.0 & 0.9 & 1530.314 & 3.684 & 0.144751 & 18.300 & dro6-48 & $x$ & $\oplus$ & + & $x$ & $\oplus$ & $\oplus$ & $x$ & $\oplus$ & $\oplus$ & $x$ & + & $\oplus$ & $x$ & & $x$ & $x$ & & $x$ \\
\hline$e 8 b$ & 0.8 & 8.0 & 0.8 & 756.641 & 4.444 & 0.113578 & & dro6-48 & & $\sim$ & & & $x$ & & & $x$ & & & $x$ & & & $x$ & & & $x$ & \\
\hline e8 & 0.6 & 8.0 & 0.8 & 771.968 & 4.444 & 0.113000 & & dro8-64 & & $\sim$ & & & $x$ & & & $x$ & & & $x$ & & & $x$ & & & $x$ & \\
\hline w4-368 & 0.4 & 7.0 & 0.9 & 1588.133 & 3.684 & 0.140816 & & dro6-48 & & + & + & & $\oplus$ & $\oplus$ & & $\oplus$ & + & & $\oplus$ & $x$ & & $\sim$ & $x$ & & $x$ & $x$ \\
\hline w2-368 & 0.2 & 7.0 & 0.9 & 1712.163 & 3.684 & 0.137622 & & dro6-48 & & + & $x$ & & + & $x$ & & $x$ & $x$ & & $x$ & $x$ & & $x$ & $x$ & & $x$ & $x$ \\
\hline ze4 & 0.2 & 6.15 & 0.4 & 354.628 & 4.393 & 0.106691 & & dro6-48 & & $x$ & & & $x$ & & & $x$ & & & $x$ & & & $x$ & & & $x$ & \\
\hline zze9 & 0.0 & 7.800001 & 0.9 & 2224.815 & 4.105 & 0.120223 & & dro8-64 & & $x$ & & & $x$ & & & $x$ & & & $x$ & & & $x$ & & & $x$ & \\
\hline ze9 & 0.0 & 7.8001 & 0.9 & 2112.079 & 4.105 & 0.120222 & & dro6-48 & & $x$ & & & $x$ & & & $x$ & & & $x$ & & & $x$ & & & $x$ & \\
\hline ns5 & 0.0 & 7.2 & 0.5 & 405.662 & 4.8 & 0.095855 & & dro8-64 & & $x$ & & & $x$ & & & $x$ & & & $x$ & & & $x$ & & & $x$ & \\
\hline s0 & 0.0 & 10.695207 & 0.708941 & 771.968 & 6.258 & 0.070830 & & dro4-32 & & $x$ & & & $x$ & & & $x$ & & & $x$ & & & $x$ & & & $x$ & \\
\hline$n-55$ & -0.5 & 10.0 & 0.5 & 505.428 & 6.667 & 0.062012 & & dro6-48 & & $x$ & & & $x$ & & & $x$ & & & $x$ & & & $x$ & & & $x$ & \\
\hline s-6 & -0.6 & 13.083066 & 0.609412 & 771.968 & 8.129 & 0.048498 & & dro4-32 & & $x$ & & & $x$ & & & $x$ & & & $x$ & & & $x$ & & & $x$ & \\
\hline wm8-631 & -0.8 & 10.1 & 0.6 & 747.545 & 6.313 & 0.066625 & & dro4-32 & & $x$ & & & $x$ & & & $x$ & & & $x$ & & & $x$ & & & $x$ & \\
\hline wm99-605 & -0.99 & 11.5 & 0.9 & 3401.251 & 6.053 & 0.072312 & & dro4-32 & & $x$ & & & $x$ & & & $x$ & & & $x$ & & & $x$ & & & $x$ & \\
\hline s-99 & -0.99 & 14.542929 & 0.534714 & 771.968 & 9.476 & 0.038531 & & dro4-32 & & $x$ & & & $x$ & & & $x$ & & & $x$ & & & $x$ & & & $x$ & \\
\hline
\end{tabular}

equatorial plane (scalar field) ${ }^{5}$ and $\Psi_{4}$ evaluated either on the $\mathrm{z}$ axis or in the equatorial plane (gravitational).

\section{QUASINORMAL-MODE MODELS AND FITS}

\section{A. Scalar-field perturbations}

\section{Perturbations at a fixed spatial position}

Consider first the case of wiggles in an individual $e^{i m \phi}$ Fourier mode of the regularized scalar field, observed at a fixed "watchpoint" spatial position in the strong-field region. We consider the model

$$
\begin{aligned}
\left(\varphi_{m}\right)_{\text {regularized }}= & B(t)+\sum_{k} A^{(k)} \exp \left(-\lambda^{(k)}\left(t-t_{\text {ref }}\right)\right) \\
& \times \sin \left(2 \pi \frac{t-t_{\text {ref }}}{P^{(k)}}+\eta^{(k)}\right),
\end{aligned}
$$

where $B$ is a spline function representing the slowly varying "background" variation of the scalar field, $k$ indexes the damped-sinusoids included in the model, $A^{(k)}, \lambda^{(k)}, P^{(k)}$, and $\eta^{(k)}$ are respectively the amplitude, damping rate, period, and phase offset of each damped-sinusoid, and the subscript ref denotes a "reference" time chosen for convenience. To avoid degeneracy between the spline and the damped-sinusoid we require that the spacing in $t$ between adjacent spline control points always be at least $1.5 P^{(\max )}$, where $P^{(\max )}:=\max _{k} P^{(k)}$ is the period of the longest-period damped-sinusoid in the model.

${ }^{5}$ Recall (cf. footnote 3 ) that in our scalar-field computations, $t$ is a Bondi-type retarded time coordinate at $\mathcal{J}^{+}$.

\section{Perturbations at the particle position}

Consider next the case of wiggles in the radiation-reaction self-force (which is implicitly defined at the particle position). This introduces two complications: the self-force is a 4-vector (with nontrivial $t, r$, and $\phi$ components for our equatorial orbits), and the field perturbation is being sampled at a timevarying position. Analogously to (18), we thus consider the model

$$
\begin{aligned}
F_{a}(u)= & \frac{B_{a}(u)}{r_{\text {particle }}^{3}(u)}+\sum_{k} \frac{A_{a}^{(k)}}{r_{\text {particle }}(u)} \exp \left(-\lambda^{(k)}\left(u-u_{\text {ref }}\right)\right) \\
& \times \sin \left(2 \pi \frac{u-u_{\text {ref }}}{P^{(k)}}-m\left(\phi_{\text {particle }}(u)-\phi_{\mathrm{ref}}\right)+\eta_{a}^{(k)}\right),
\end{aligned}
$$

where we now parametrize the particle motion using the retarded time coordinate $u,{ }^{6} B_{a}$ is now a 4 -vector spline function representing the background variation of the self-force along the particle worldline, $k$ again indexes the damped-sinusoids included in the model, $A_{a}^{(k)}, \lambda^{(k)}$, $P^{(k)}$, and $\eta_{a}^{(k)}$ are now respectively the 4-vector amplitude, damping rate, period, and 4-vector phase offset of each damped-sinusoid, and the subscript ref again denotes a "reference" time chosen for convenience. The nondegeneracy

\footnotetext{
${ }^{6}$ Heuristically, the choice of $u$ rather than $t$ as an independent variable in the model is suggested by the QNM signals propagating outward along light cones after being excited near periapsis. However, it is not clear that this is a good approximation to actual QNM dynamics, so we experimented with models using both $t$ and $u$ as independent variables. We found the latter to give better fits to our numerical data.
} 
TABLE III. This table explains the meanings of the "wiggle symbols" used in Table II and Fig. 1.

\begin{tabular}{ll}
\hline \hline Symbol & \multicolumn{1}{c}{ Meaning } \\
\hline$\oplus \quad \begin{array}{l}\text { we observed oscillations in the diagnostics, successfully fit the appropriate wiggle model described in Sec. IV A over a } t \text { or } u \\
\text { range of } \geqslant 4 \text { wiggle periods, and performed the Monte Carlo error analysis described in Sec. IV A } 5 .\end{array}$ \\
$+\quad \begin{array}{l}\text { we observed oscillations in the diagnostics and successfully fit the appropriate wiggle model described in Sec. IV A, but the } \\
\text { model was fitted over too short a } t \text { or } u \text { range ( }<4 \text { wiggle periods) for the Monte Carlo error analysis described in Sec. IV A } 5 \\
\text { to be used }\end{array}$ \\
$\begin{array}{l}\text { we observed oscillations in the diagnostics which visually appeared to be wiggles, with physically reasonable period and } \\
\text { decay rate, but we did not attempt to quantitatively fit a wiggle model }\end{array}$ \\
$\begin{array}{l}\text { we observed oscillations in the diagnostics which might have been wiggles, but these oscillations were not clearly separated } \\
\text { from the background variation in the diagnostics }\end{array}$ \\
$\begin{array}{l}\text { we did not observe wigglelike oscillations in the diagnostics; this could mean either that no wiggles are present, or } \\
\text { alternatively that wiggles were present but they were at too low an amplitude and/or insufficiently separated from the } \\
\text { background variation to be observed } \\
\text { diagnostics were not computed, not computed sufficiently accurately for studying wiggles, or were computed but not assessed }\end{array}$ \\
(blank)
\end{tabular}

condition on the background spline now applies to the spacing in $u$ between adjacent spline control points.

Notice that the damping rate and oscillation period of each damped-sinusoid are common across all tensor components of the self-force. The $-m \phi_{\text {particle }}(u)$ term models the variation in oscillation phase due to particle (i.e., the sampling point) motion in $\phi$. The $1 / r_{\text {particle }}^{3}(u)$ and $1 / r_{\text {particle }}(u)$ factors model the expected far-field variation in self-force and in the oscillation eigenfunction amplitude with position. (Actual QNM eigenfunctions have a much more complicated variation of amplitude with spatial position, but for simplicity, we omit this from our model.)

\section{Perturbations at $\mathcal{J}^{+}$}

Finally, consider the case of wiggles in the radiated (physical) field at $\mathcal{J}^{+}$. Because of the hyperboloidal time slices, we observe these at finite coordinate time $t$ (the $\mathcal{J}^{+}$time has an arbitrary offset relative to the strong-field coordinate time). As noted in Sec. III, here it is somewhat difficult to separate wiggles from the overall radiation pattern, so we consider second time derivatives of the physical scalar-field modes.

TABLE IV. This table gives the resolution in $r_{*}$ and $\theta$ of the coarsest and finest grids for each of our standard grid structures. The finest grid covers a neighborhood of the worldtube in each slice, while successively coarser grids cover correspondingly larger regions using standard Berger-Oliger 2:1 mesh refinement.

\begin{tabular}{lllllc}
\hline \hline & \multicolumn{2}{c}{ Coarsest grid } & & \multicolumn{2}{c}{ Finest grid } \\
\cline { 2 - 3 } \cline { 6 - 6 } & $\begin{array}{l}R_{*} \\
(M)\end{array}$ & $\begin{array}{c}\theta \\
\text { (radians) }\end{array}$ & & $\begin{array}{c}R_{*} \\
(M)\end{array}$ & $\begin{array}{c}\theta \\
\text { (radians) }\end{array}$ \\
\hline dro12-96 & $1 / 12$ & $\pi / 216$ & & $1 / 96$ & $\pi / 1728$ \\
dro10-80 & $1 / 10$ & $\pi / 180$ & & $1 / 80$ & $\pi / 1440$ \\
dro8-64 & $1 / 8$ & $\pi / 144$ & & $1 / 64$ & $\pi / 1152$ \\
dro6-48 & $1 / 6$ & $\pi / 108$ & & $1 / 48$ & $\pi / 864$ \\
dro4-32 & $1 / 4$ & $\pi / 72$ & & $1 / 32$ & $\pi / 576$ \\
\hline \hline
\end{tabular}

Analogously to (18) and (19), we thus consider the model

$$
\begin{aligned}
\left.\partial_{t t}\left(\left(\varphi_{m}\right)_{\text {regularized }}\right)\right|_{\mathcal{J}^{+}}= & B(t)+\sum_{k} A^{(k)} \exp \left(-\lambda^{(k)}\left(t-t_{\text {ref }}\right)\right) \\
& \times \sin \left(2 \pi \frac{t-t_{\text {ref }}}{P^{(k)}}+\eta^{(k)}\right)
\end{aligned}
$$

where the meanings of all terms (and the nondegeneracy condition on the background spline) are the same as in (18).

\section{Fitting the models}

For each of the models (18), (19), and (20), we visually inspect plots of our time-series data to identify a suitable range of the independent variable for fitting and to choose initial guesses for the background and wiggle parameters, then use the nonlinear least-squares fitting subroutine LMDIF1 from the MINPACK library [68] to fit the model to the data. To make the model closer to linear (which improves the convergence of the nonlinear fitting), we fit the wiggle amplitudes and phases as cosine- and sine-component amplitudes [i.e., $A \sin (X+\eta)$ is actually fitted as $\left.A^{(\cos )} \cos (X)+A^{(\sin )} \sin (X)\right]$. In most cases, we used uniform weighting for the fits, but in a few cases we used weights proportional to $r^{3}$ so as to improve the fit at late times (close to apoapsis).

\section{Uncertainties in the fitted wiggle parameters}

The residuals from our wiggle fits are not random, but rather are dominated by low-amplitude oscillations of similar frequency to the wiggles themselves (this can be seen in Figs. 4 and 5). This means that formal uncertainty estimates for the fitted parameters $\left(P^{(k)}, \tau^{(k)}\right)$ (derived assuming uncorrelated Gaussian residuals) are not realistic. Because of the oscillatory nature of the residuals, the fitted parameters are slightly dependent on the precise choice of fitting interval; this is, in fact, usually the dominant uncertainty in the fitted parameters.

We use a Monte Carlo procedure to estimate realistic uncertainties in the fitted parameters: Given a fit of one of the above wiggle models to our data in some interval $I$ (in either 


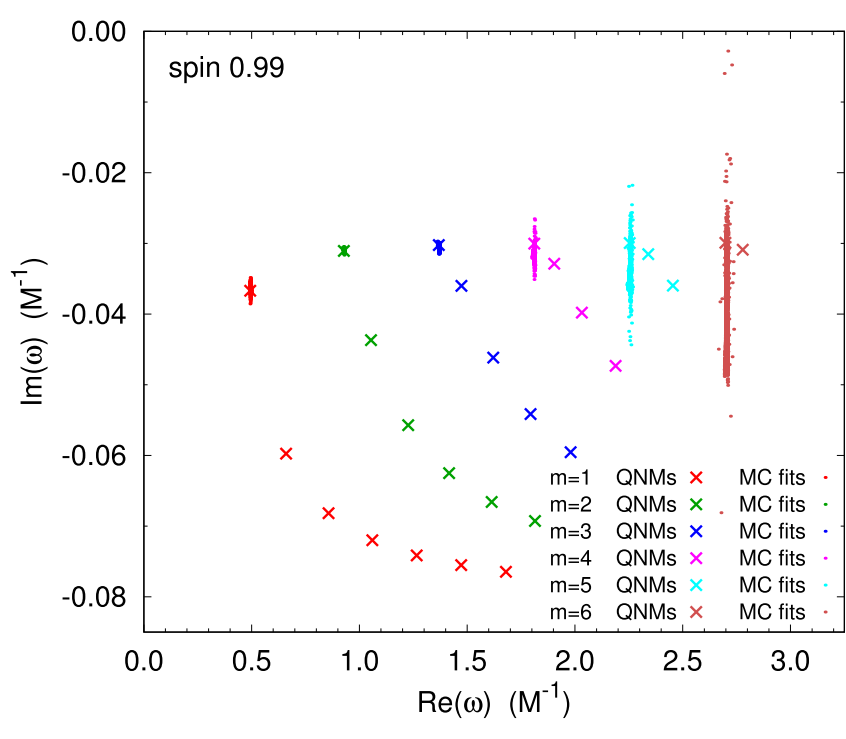

FIG. 6. This figure shows all the Kerr QNM frequencies in the region $(\operatorname{Re}(\omega), \operatorname{Im}(\omega)) \in([0,3.25] \times[-0.085,0]) M^{-1}$ for Kerr spin $\tilde{a}=0.99$, together with our fitted complex frequencies' Monte Carlo error estimates for the $m=1$ through $m=6$ modes of the $(\tilde{a}, p, e)=(0.99,3,0.8)$ configuration. For $m=2$ and 3 , the Monte Carlo points are all very close to, and visually almost hidden by, the least-damped QNM's $\mathbf{x}$ symbol. The apparent anisotropy of the Monte Carlo "point clouds" in this plot is a visual illusion due to the anisotropic plot scale; the point clouds are actually approximately isotropic in $(\operatorname{Re}(\omega), \operatorname{Im}(\omega))$. Some of these QNM frequencies and all of the Monte Carlo error estimates are also plotted at different scales in Fig. 7.

$t$ or $u$ ) of length $L_{\mathrm{fit}} \geqslant 4 P^{(\max )}$, we randomly choose $N_{\text {trial }}=$ 300 subintervals of $I$ (randomly sampling each lower and upper interval endpoint from a uniform distribution) subject to the constraint that each subinterval must have a minimum length of $L_{\min }=3 P^{(\max )}{ }^{7}$ Then we repeat the wiggle-model fit for each subinterval. ${ }^{8}$ The ensemble of the $N_{\text {trial }}$ sets of Monte-Carlo-trial fitted parameters then provides an estimate of the uncertainty in the fitted parameters from the fullinterval fit.

After allowing initial transients to decay, our numerical calculations extend over a number of particle orbits. Because the particle orbit precesses strongly, each orbit places the particle in a different position with respect to any fixed watchpoint or $\mathcal{J}^{+}$observer. For each orbit we repeat the entire fitting procedure (including the full set of Monte Carlo subinterval trials). Our final estimate for the uncertainty in the fitted

\footnotetext{
${ }^{7}$ The minimum-length requirement for the subintervals ensures that each subinterval is long enough to allow a reasonable estimate of the wiggle decay rate and period (the fitting errors should scale roughly inversely with $L_{\min }$ ). The minimum-length requirement for the full fitting interval ensures that different subintervals can sample significantly different regions of the data.

${ }^{8}$ Each subinterval fit uses a subset of the original fit's background spline control points which just span that subinterval, plus one point outside the subinterval on each of the interval's left and right endpoints.
}

parameters is obtained from the union of all the Monte Carlo trials over several (typically 3 ) distinct orbits.

This procedure has two main limitations: (1) The procedure is not applicable to cases where the overall fitting interval is too short (length $L_{\mathrm{fit}}<4 P^{(\max )}$ ). (Footnote 7 outlines the reasons for this.) (2) If a wiggle is rapidly damped, then the wiggle amplitude becomes very small at the right (large $t$ or $u$ ) end of a long fitting interval, so a subinterval of near-minimum length $\left(3 P^{(\max )}\right)$ which is close to the right end of the overall fitting interval will have a poorly constrained fit, yielding a large scatter in the fitted parameters.

These limitations are most severe when the wiggles have low amplitude and are rapidly damped, as is the case for low Kerr spins.

\section{Other error sources}

There are a number of other error sources not taken into account in our Monte Carlo error estimates:

(1) Our (TW's) numerical code only computes the diagnostics to finite accuracy. Comparing diagnostics between calculations done with different numerical resolutions, we have generally excluded any data where the diagnostic computed at our highest resolution (that shown in Table II) differs from that computed at the next-lower resolution by more than a few percent.

(2) Wiggles are not perfectly separable from the background variation of the diagnostics. That is, the actual frequency spectra of the diagnostics are almost certainly continuous, and cannot be unambiguously separated into lowfrequency (background) and high-frequency (wiggle) components.

(3) Our models for the background variation are imperfect. Our constraint that background spline control points must be spaced at least 1.5 wiggle periods apart keeps the background and wiggles from being degenerate, but at the cost of leaving the background model unable to accurately fit some nonwiggle variations, particularly for small- $m$ (longer-period) wiggles where the spline control points are forced to be quite far apart.

(4) For wiggles in $F_{a}$, our wiggle model (19) does not accurately include the actual spatial variation of the wiggle (QNM) eigenfunctions.

(5) There may be multiple wiggle modes present simultaneously in the diagnostics for a single $m$. Although our wiggle models and fitting software support simultaneously fitting an arbitrary number of wiggles, we have generally not done this, i.e., we have generally only attempted to fit a single-wiggle model for each diagnostic time series. ${ }^{9}$

We believe that all these other error sources are small, but it is difficult to quantify them.

For each wiggle fit, we visually assess the fit residuals to look for obvious systematics. For all results reported here the fit residuals are at least a factor of 10 smaller than the wiggle

\footnotetext{
${ }^{9}$ In a few cases where our best-fitting single-wiggle model residuals showed strong systematics, we then proceeded to fit two-wiggle models. These improved the residuals by at least an order of magnitude. However, all the results presented in Sec. V A are based on single-wiggle fits.
} 

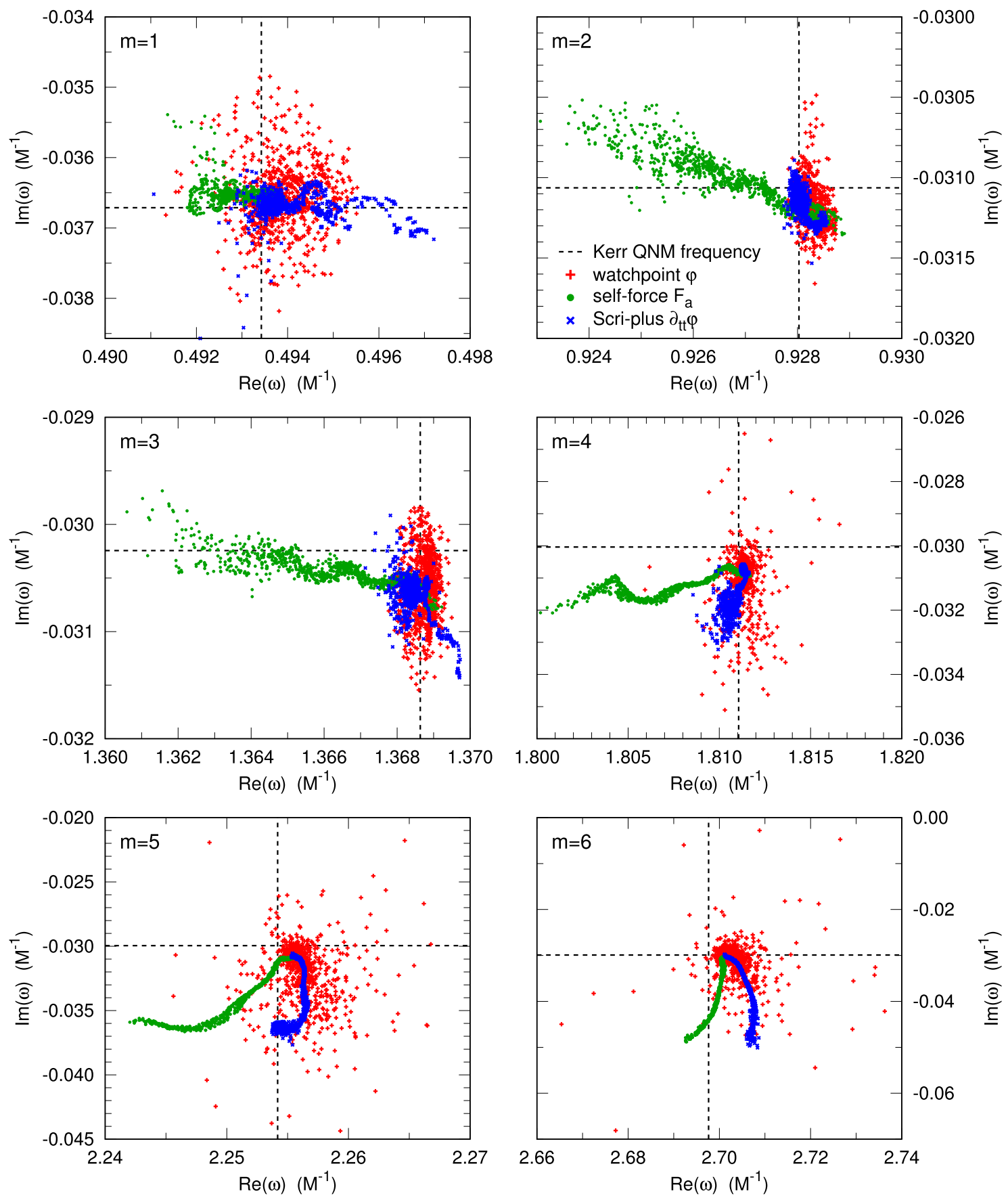

FIG. 7. This figure shows the least-damped Kerr spin $\tilde{a}=0.99$ QNM frequencies for $m=1$ through $m=6$, together with our fitted complex frequencies' Monte Carlo error estimates for the $m=1$ through $m=6$ modes of the $(\tilde{a}, p, e)=(0.99,3,0.8)$ configuration. In each subfigure, the dashed lines intersect at the least-damped QNM frequency. The legend is common to all of the subfigures. These same Monte Carlo error estimates are all plotted at a common scale in Fig. 6; in comparison, this figure zooms in on a small region around the least-damped QNM for each $m$.

amplitude; in most cases they are a factor of 30 to 100 smaller. This suggests that our fits are indeed accurately modeling at least the dominant wiggle features of the diagnostics.

\section{B. Gravitational perturbations}

In the gravitational case, we search for the QNM frequencies in the individual (spheroidal) $(\mathfrak{l} m)$ modes. By looking at individual $(\mathfrak{l} m)$-modes we minimize the number of
QNMs that need to be modelled (fitted) simultaneously. Since QNMs appear naturally as spheroidal modes, using spheroidal modes minimizes the amount of "crosstalk" mixed in from neighboring modes. Note that since the spheroidicity of the spheroidal harmonics in the Teukolsky equation depends on the frequency of the modes, the QNMs have complex spheroidicity and will not project perfectly on the corresponding (real) spheroidal modes that appear in the field solutions. Consequently, "crosstalk" between the modes cannot be fully 
eliminated. Nonetheless, the crosstalk in the spheroidal modes should be significantly smaller than if one were to use the spherical $\ell$-modes.

\section{Fit models}

The fit models used in the gravitational case are very similar to the ones used in the scalar case. For the "watchpoint" diagnostics, we use

$$
\begin{aligned}
\Phi_{-2}^{(\mathrm{l} m)}= & \sum_{n} B^{(n)} t^{n}+\sum_{k}\left\{A_{s}^{(k)} \sin \left[\omega_{k}\left(t-t_{\mathrm{ref}}\right)\right]\right. \\
& \left.+A_{c}^{(k)} \cos \left[\omega_{k}\left(t-t_{\mathrm{ref}}\right)\right]\right\} e^{-\alpha_{k}\left(t-t_{\mathrm{ref}}\right)} .
\end{aligned}
$$

In this case, the smooth background of the signal is modelled by a simple polynomial in $t$. We maximize the number of linear fit parameters by writing the model as a sum of sines and cosines.

Similarly, the (lm) modes contributing to the local gravitational self-force are modelled by

$$
\begin{aligned}
F_{a}^{(\mathrm{l} m)}= & \sum_{n} B_{a}^{(n)} u^{n}+\sum_{k}\left\{A_{s, a}^{(k)} \sin \left[\omega_{k}\left(u-u_{\mathrm{ref}}\right)-m \phi_{\text {particle }}\right]\right. \\
& \left.+A_{c, a}^{(k)} \cos \left[\omega_{k}\left(u-u_{\mathrm{ref}}\right)-m \phi_{\text {particle }}\right]\right\} \frac{e^{-\alpha_{k}\left(u-u_{\mathrm{ref}}\right)}}{r_{\text {particle }}} .
\end{aligned}
$$

As in the scalar case, the main shortcoming in this model is the inaccurate modeling of the QNMs' radial profiles.

Finally, the model for the gravitational waveform at $\mathcal{J}^{+}$is very similar to the model for the watchpoints,

$$
\begin{aligned}
\lim _{r \rightarrow \infty} r \Psi_{4}^{(\mathrm{l} m)}= & \sum_{n} B^{(n)} u^{n}+\sum_{k}\left\{A_{s}^{(k)} \sin \left[\omega_{k}\left(u-u_{\mathrm{ref}}\right)\right]\right. \\
& \left.+A_{c}^{(k)} \cos \left[\omega_{k}\left(u-u_{\mathrm{ref}}\right)\right]\right\} e^{-\alpha_{k}\left(u-u_{\mathrm{ref}}\right)} .
\end{aligned}
$$

\section{Fitting procedure}

The only nonlinear parameters in the above models are the QNM frequencies $\omega_{k}$ and decay constants $\alpha_{k}$. Consequently, for fixed $\omega_{k}$ and $\alpha_{k}$ the remaining parameters can be determined efficiently through a linear least squares procedure. This is implemented by using MATHEMATICA's LinearModelFit routine for each diagnostic on a suitable time window of data. To reduce the impact of unmodelled higherorder QNMs, these fits are weighted by $\exp \left(2 \alpha_{1} t\right)$. Typically, the fits include around 20 terms in the background model and up to eight QNMs.

The $\omega_{k}$ and $\alpha_{k}$ are then determined by maximizing the sum of the adjusted $R^{2}$ values of all the component fits. This is implemented using MATHEMATICA's FindMaximum with the PrincipleAxis method. The initial values for $\omega_{k}$ and $\alpha_{k}$ are set by the numerically known corresponding QNMs offset by a random $\mathcal{O}(1 \%)$ amount. An indication of the modelling error is obtained by varying the fit window and number of background terms, and determining the spread of the best fits.

\section{DATA AND QUASINORMAL-MODE FITS}

\section{A. Scalar field}

We have surveyed a large number of configurations for Kerr spin $\tilde{a}=0.99$, together with a smaller number of configurations for other Kerr spins; for selected configurations, we have fitted (or attempted to fit) wiggle models as described in Sec. IV A. Tables II and III describe all the configurations surveyed here, and Fig. 1 shows the (periapsis radius, orbital eccentricity) phase space of the $\tilde{a}=0.99$ configurations.

Figures 2 and 3 show the wiggles in the scalar-field diagnostics for the $(\tilde{a}, p, e)=(0.99,3,0.8)$ configuration, for $m=1$ and $m=4$, respectively. Notice that the wiggles are visible in all the field diagnostics. Notice also the much higher frequency and smaller amplitude of the $m=4$ wiggles.

Figures 4 and 5 show our model fits to these wiggles for $m=1$ and $m=4$, respectively. Notice that in each case the spline control points span a wider range of $t$ or $u$ than the range over which the model is fitted. The $y$ coordinates at the spline control points outside the model-fitting range are still adjusted by the least-squares fitting algorithm, but have only small influences on the model within the fitting range.

Figures 6-8 show the fitted complex frequencies and their Monte Carlo error estimates, compared to Kerr QNM frequencies calculated by Berti, Cardoso, and Starinets [69-71]. In each case, the fitted frequencies agree with the calculated QNM frequencies, lending further support to the identification of wiggles with QNMs (more precisely, QNMs sampled at the observation points).

\section{B. Gravitational field}

We now turn our attention to gravitational perturbations. We first consider the same $(\tilde{a}, p, e)=(0.99,3,0.8)$ configuration studied in Figs. 2-7 in the scalar field case. Figure 9 displays both the gravitational self-force at the particle location and the waveform observed at $\mathcal{J}^{+}$. When looking at the local self-force the wiggles are most pronounced in the $F_{\phi}$ component. However, faint traces of wiggles can be found by zooming in on the $F_{t}$ and $F_{r}$ components. We note that the relative amplitudes of the wiggles in the gravitational self-force are much smaller than those in the scalar case for the same orbit (shown in Figs. 2 and 3).

The waveform observed at $\mathcal{J}^{+}$depends on the viewing angle. When the system is viewed "face on" (middle panel of Fig. 9) the waveform is determined by the $m=2$ modes with the $m=\mathfrak{l}=2$ dominating. In this case, the wiggles appear as a clear exponentially damped sinusoid. When the system is viewed "edge on" (bottom panel of Fig. 9), the wiggles have a much more irregular shape, consistent with a much larger collection of $m$ and $\mathfrak{l}$ contributing to the wiggles. Also note that while the overall waveform has a much larger amplitude when viewed edge on (due to contributions from higher modes), the observed wiggles are actually stronger when the system is viewed "face on." This is consistent with the wiggles being dominated by the $\mathfrak{l}=m=2$ mode.

One of the advantages of using a frequency domain approach is that we can easily isolate individual $\mathrm{l} m$-modes (as defined in Sec. II B). Figure 10 shows different aspects of the $\mathfrak{l}=m=2$ mode of the gravitational perturbation generated by a particle on our standard $(\tilde{a}, p, e)=(0.99,3,0.8)$ configuration. The $\mathfrak{l}=m=2$ mode of the gravitational self-force (top panel) shows the same qualitative features as the full GSF; the $F_{\phi}$ components show the most obvious wiggles with 

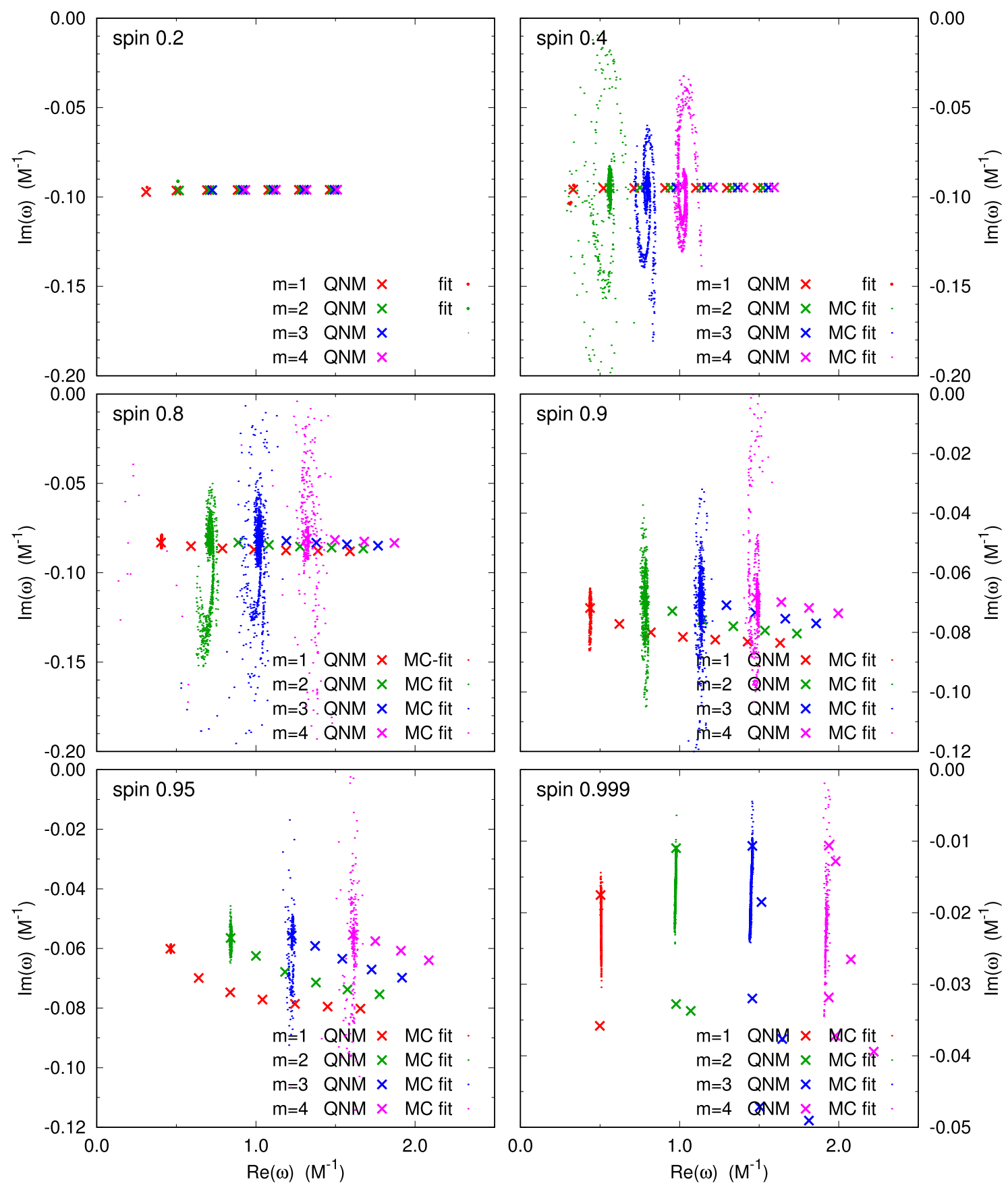

FIG. 8. This figure shows all the Kerr QNM frequencies in the plot regions, together with our fitted complex frequencies’ Monte Carlo error estimates for the $m=1$ through $m=4$ modes of the all the Kerr spin $\tilde{a} \neq 0.99$ configurations. (This is analogous to Fig. 6 for $\tilde{a} \neq 0.99$.) The apparent anisotropy of the Monte Carlo "point clouds" in this plot is a visual illusion due to the anisotropic plot scales; the point clouds are actually approximately isotropic in $(\operatorname{Re}(\omega), \operatorname{Im}(\omega))$.

weak wiggles visible in the other components. The $\mathfrak{l}=m=2$ mode of the field observed at $\mathcal{J}^{+}$shows a clean exponentially decaying sinusoid wiggle just as the full field. In addition, the bottom panel of Fig. 10 shows the local Teukolsky variable $\Phi_{-2}^{(22)}$ at two watch points located on the background Kerr spacetime's symmetry axis at radii corresponding to the periapsis and apoapsis of the particle orbit. These show the cleanest wiggles of any of our diagnostics.

To test our hypothesis that the observed wiggles are, in fact, QNM excitations, we perform a global fit of our three field diagnostics (local gravitational self-force, field at $\mathcal{J}^{+}$, and field at watchpoints) following the methodology set out in Sec. IV B. Table V summarizes the results for some low order $\mathrm{l} m$ modes. In each, case we recover the principal QNM frequency and damping time of the gravitational field within the estimated numerical precision of the fits. This provides yet more evidence for our hypothesis that the observed wiggles are QNM excitations. Note that while our fits include multiple QNMs, we do not conclusively recover any of the modes beyond the principal mode. (More precisely, we find that the 

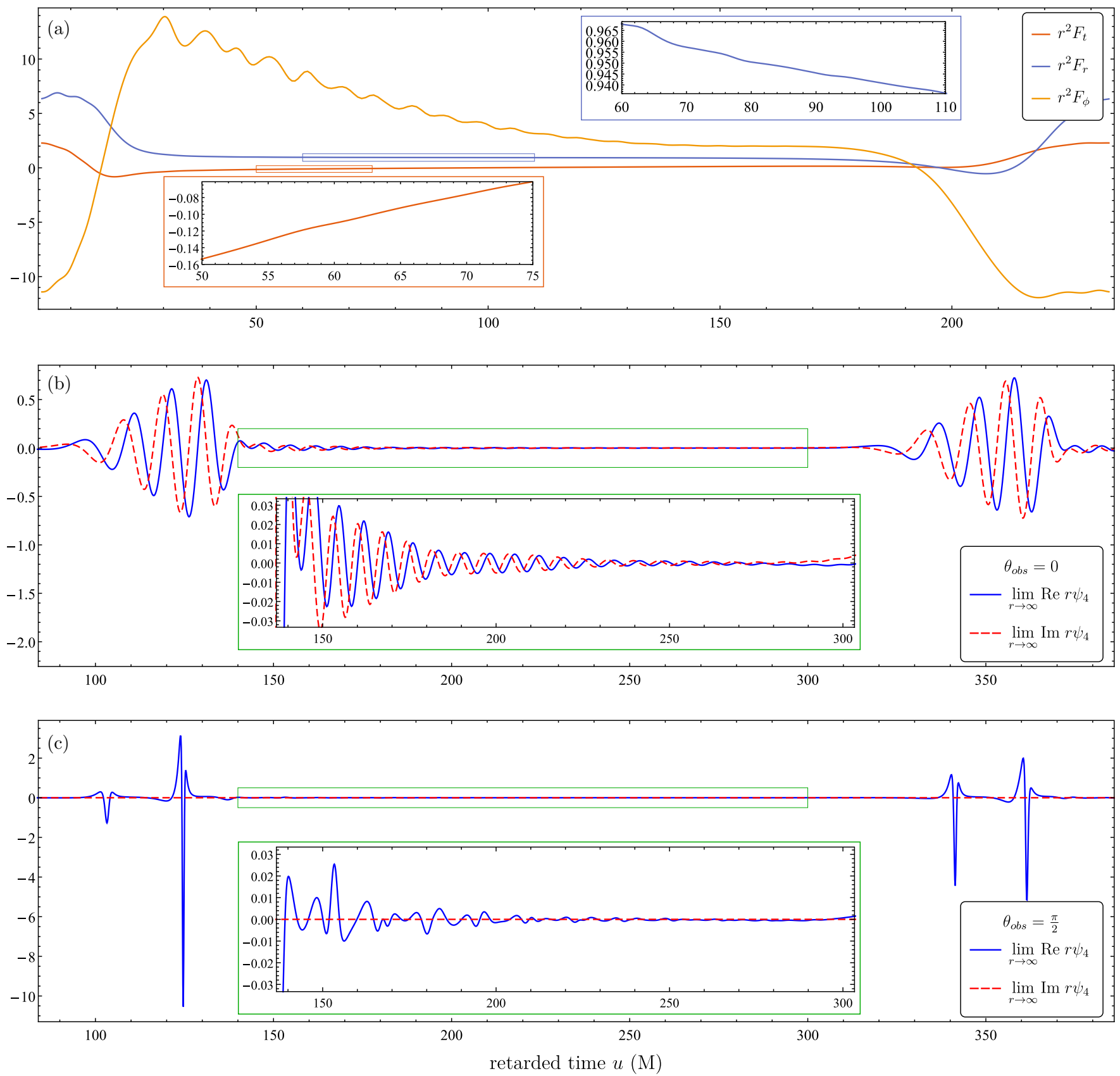

FIG. 9. Three different aspects of the gravitational perturbation for the ( $\tilde{a}, p, e)=(0.99,3,0.8)$ configuration. (a) The three nonzero components of the gravitational self-force at the particle. The $F_{\phi}$ component has very prominent wiggles. The wiggles in the other two components are present but only visible after zooming in. (b) The value of $\psi_{4}$ observed at $\mathcal{J}^{+}$when the system is viewed "face on" $\left(\theta_{\text {observer }}=0\right)$. We observe very clean exponentially decaying wiggles right up to the next burst produced when the particle approaches the central black hole again. (c) The value of $\psi_{4}$ observed at $\mathcal{J}^{+}$when the system is viewed "edge on" $\left(\theta_{\text {observer }}=\pi / 2\right)$. In this case, the wiggle pattern is much more complex as multiple $(\mathfrak{l}, m)$ modes contribute.

estimated numerical errors of the recovered complex frequencies are comparable to the variation of the initial seed for the optimization.) Not including the higher modes, however, led to observable bias in the recovery of the principal QNMs.

\section{Dependence on orbital parameters}

In this section, we study how the strength of the QNM excitations in the gravitational field depends on the parameters of the orbit. For this investigation, we leverage the ease with which the $(\mathfrak{l} m)$ modes of the gravitational field can be computed using our frequency domain code. As a measure of the strength of the excitations we take the amplitudes $A_{c}^{(k)}$ and $A_{s}^{(k)}$ in (23), which we combine to define

$$
\left|A^{(k)}\right|:=\sqrt{\left(A_{c}^{(k)}\right)^{2}+\left(A_{s}^{(k)}\right)^{2}} .
$$

For the purpose of this investigation we assume that the observed wiggles are indeed QNM excitations, we therefore determine the $\left|A^{(k)}\right|$ by a linear fit, keeping the frequencies 

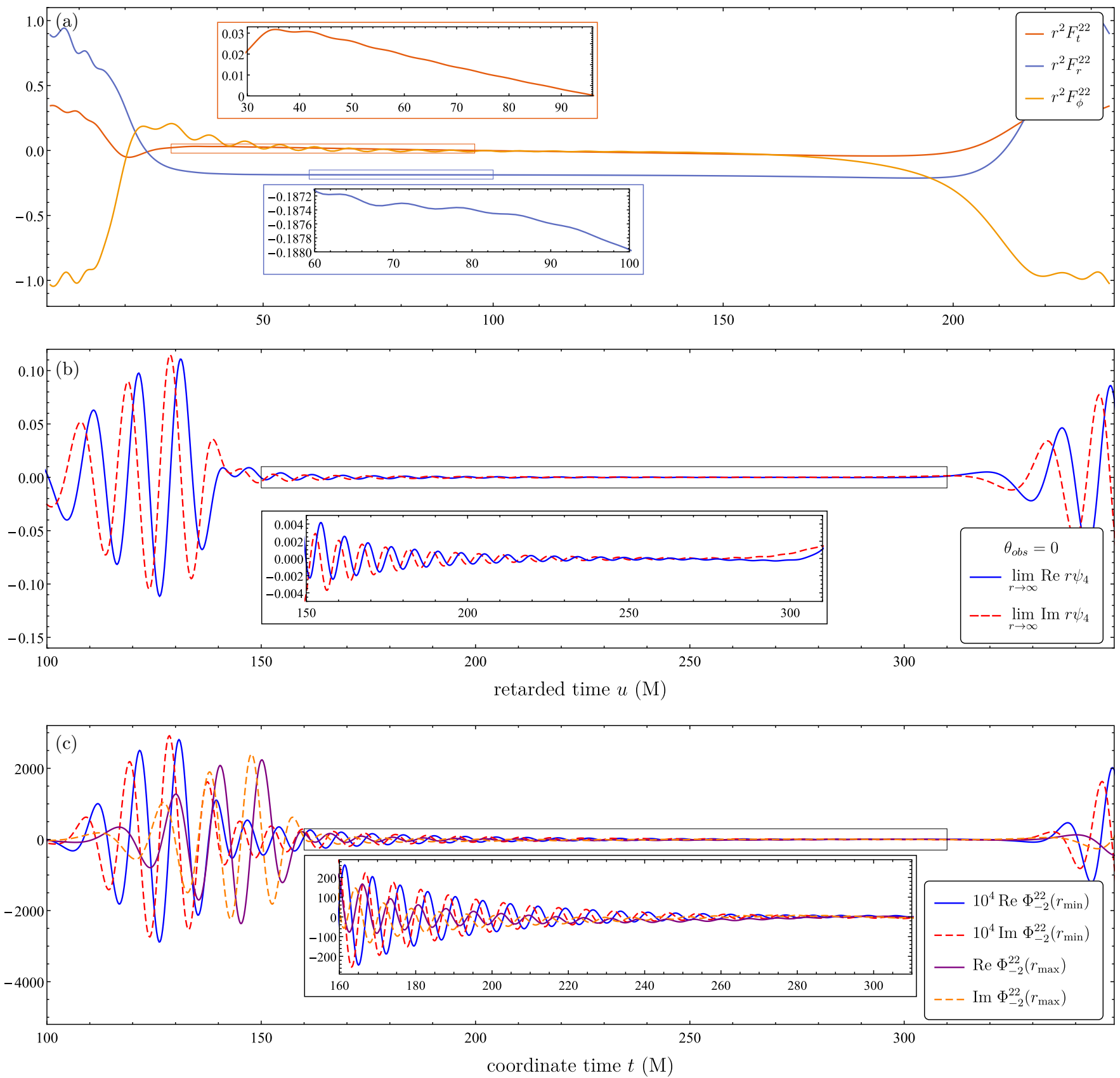

FIG. 10. In this figure, we focus on the $\mathfrak{l}=m=2$ mode of the gravitational perturbation for the $(\tilde{a}, p, e)=(0.99,3,0.8)$ configuration. (a) The three nonzero components of the gravitational self-force. (b) The value of $\psi_{4}$ observed at $\mathcal{J}^{+}$when the system is viewed "face on" $\left(\theta_{\text {observer }}=0\right)$. (c) The Teukolsky variable $\Phi_{-2}^{(22)}$ evaluated on the symmetry axis $\theta=0$ and at two different radii $r=r_{\max }$ and $r=r_{\min }$.

and decay rates fixed at the exact QNM values. The value of $\left|A^{(k)}\right|$ depends on the choice of $u_{\text {ref }}$ in (23). In this section, we choose $u_{\text {ref }}$ to coincide with the particle passing through either the periapsis or apoapsis of the orbit.

We are particularly interested in whether excitation of the QNMs exhibits a strong dependence on the alignment of the discrete orbital frequency spectrum of the orbit with the QNM mode. If strong localized "resonances" between the orbit frequency spectrum and QNM excitations were to exist, these could have significant impact on waveform modeling strategies, as they would hamper an attempt to apply reduced order modeling to build efficient waveforms. On the other hand, such a phenomenon might lead to interesting and rich dynamics. To quantify the alignment between a QNM and the particle orbit, we define

$$
\delta^{(k)}:=\min _{n \in \mathbb{Z}} \frac{\left|\omega^{(k)}-m \Omega_{\phi}-n \Omega_{r}\right|}{\Omega_{r}},
$$

i.e., $\delta^{(k)}$ is the distance between the QNM frequency $\omega^{(k)}$ and the nearest line in the orbit frequency spectrum, normalized such that $\delta^{(k)}=0$ corresponds to maximal alignment and $\delta^{(k)}=1 / 2$ to maximal misalignment.

In Fig. 11, we examine the dependence of the QNM amplitude (for the least-damped $(k=1) \mathfrak{l}=m=2$ QNM) on the spin $a$ of the background Kerr spacetime while keep- 
TABLE V. Numerical fits of the wiggles in the gravitational (spheroidal) $(\mathfrak{l} m)$-mode field diagnostics for the $(\tilde{a}, p, e)=$ $(0.99,3,0.8)$ configuration. In each row, the top values are the fitted frequency/decay rate; the bottom values are the known values for (least damped) gravitational QNMs. Fitting errors are indicated in parentheses, e.g., 0.870891(7) means $0.870891 \pm 7 \times 10^{-6}$.

\begin{tabular}{|c|c|c|c|}
\hline $\mathfrak{l}$ & $m$ & $\omega_{\mathfrak{l} m k}$ & $\alpha_{\mathfrak{l} m k}$ \\
\hline \multirow{2}{*}{2} & \multirow{2}{*}{0} & $0.421(3)$ & $0.069(4)$ \\
\hline & & 0.423 & 0.072 \\
\hline \multirow{2}{*}{2} & \multirow{2}{*}{1} & $0.57278(6)$ & $0.04624(5)$ \\
\hline & & 0.57274 & 0.04620 \\
\hline \multirow{2}{*}{3} & \multirow{2}{*}{1} & $0.7891(1)$ & $0.0618(3)$ \\
\hline & & 0.7891 & 0.0618 \\
\hline \multirow{2}{*}{2} & \multirow{2}{*}{2} & $0.870891(7)$ & $0.029386(4)$ \\
\hline & & 0.870892 & 0.029390 \\
\hline \multirow{2}{*}{3} & \multirow{2}{*}{2} & $0.999142(9)$ & $0.037128(6)$ \\
\hline & & 0.999140 & 0.037129 \\
\hline \multirow{2}{*}{4} & \multirow{2}{*}{2} & $1.1771(1)$ & $0.0510(4)$ \\
\hline & & 1.1770 & 0.0511 \\
\hline \multirow{2}{*}{3} & \multirow{2}{*}{3} & $1.32308(3)$ & $0.02940(1)$ \\
\hline & & 1.32308 & 0.02940 \\
\hline \multirow{2}{*}{4} & \multirow{2}{*}{3} & $1.43371(3)$ & $0.03323(3)$ \\
\hline & & 1.43372 & 0.03323 \\
\hline \multirow{2}{*}{5} & \multirow{2}{*}{3} & $1.58353(3)$ & $0.04306(3)$ \\
\hline & & 1.58351 & 0.04308 \\
\hline \multirow{2}{*}{4} & \multirow{2}{*}{4} & $1.7731(2)$ & $0.02946(6)$ \\
\hline & & 1.7731 & 0.02947 \\
\hline \multirow{2}{*}{5} & \multirow{2}{*}{4} & $1.87254(4)$ & $0.03160(5)$ \\
\hline & & 1.87254 & 0.03160 \\
\hline \multirow{2}{*}{5} & \multirow{2}{*}{5} & $2.2216(2)$ & $0.0295(1)$ \\
\hline & & 2.2217 & 0.0295 \\
\hline
\end{tabular}

ing eccentricity and ratio of the orbital frequencies [matching the eccentricity and frequency ratio for the $(\tilde{a}, p, e)=$ $(0.99,3,0.8)$ configuration]. We see that the dependence of the QNM excitation amplitude on the Kerr spin $a$ is very smooth, with no noticeable dependence on the spectrum misalignment parameter $\delta$. Notice that the QNM excitations persist for negative spins (i.e., retrograde orbits), although they become exceedingly weak.

Figure 12 explores the dependence of the amplitude $\left|A^{(1)}\right|$ of the least damped $(k=1) \mathfrak{l}=m=2$ QNM on the particle orbital eccentricity. For this exploration, we keep the spin of the background Kerr spacetime fixed at $a=0.95 M$, and we fix the periapsis distance at $r_{\min }=1.85 M$. The relationship between $\left|A^{(1)}\right|$ and $e$ appears almost linear by eye, with slight deviations both at high and low eccentricity. If we subtract off the dominant trend in the form of a quintic fit in $e$, we see what appears to be a systematic trend where orbits with $\delta^{(1)}=0$ have a slightly larger amplitude $\left|A^{(1)}\right|$ than orbits with $\delta^{(1)}=1 / 2$. This difference becomes stronger for low eccentricity orbits. This latter effect is consistent with the frequency spectrum of the orbit becoming sparser at lower eccentricities. However, we stress that this effect is very small, with the variation of the QNM amplitude $\left|A^{(1)}\right|$ due to changing $\delta^{(1)}$ being only about 1 part in $10^{3}$.

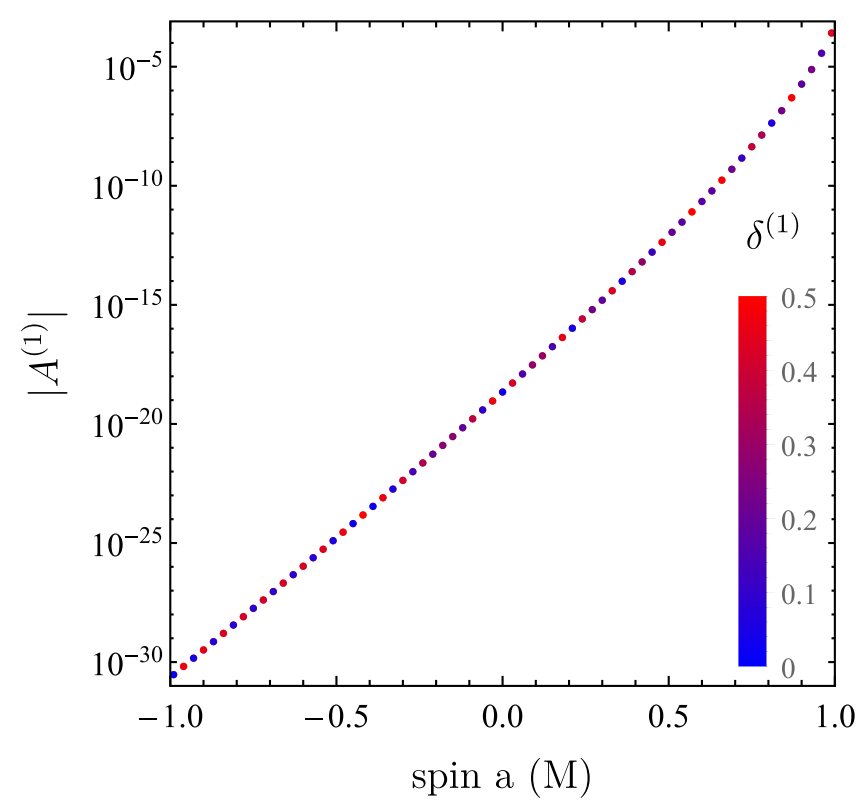

FIG. 11. Dependence of the the fitted QNM amplitude $\left|A_{1}\right|$ of the lowest damped $\mathfrak{l}=m=2$ QNM as a function of the primary spin $a$ for a sequence of orbits with fixed $e=0.8$ and ratio $\Omega_{\phi} / \Omega_{r}=2.684694379 \ldots$ [the frequency ratio for the $(\tilde{a}, p, e)=$ $(0.99,3,0.8)$ configuration]. The parameter $u_{\text {ref }}$ is fixed to coincide with the particle passing through apoapsis. The data points are shaded according to the degree of alignment $\delta$ of the particle spectrum with the QNM frequency $\omega^{(1)}$.

Finally, Fig. 13 explores the relation between the amplitude $\left|A^{(1)}\right|$ of the least damped $(k=1) \mathfrak{l}=m=2$ QNM and the particle inverse periapsis distance $M / r_{\min }$, keeping the Kerr spin $(a=0.95 M)$ and particle eccentricity $(e=0.8)$ fixed. As is to be expected, the amplitude $\left|A^{(1)}\right|$ drops off sharply as we increase the particle periapsis radius.

We emphasize that the overall shape of the plots in Figs. 11-13 depends sensitively on the choice of $u_{\text {ref }}$, hence one should not read too much into the shapes themselves. However, there are three main lessons that we learn from this investigation that do not depend on the choice of $u_{\text {ref }}$. (1) The amplitudes of the wiggles depend smoothly on the Kerr spin (Fig. 11) and orbital parameters (Figs. 12 and 13). In particular, no fine-tuning is needed for wiggles to appear. (2) The wiggles are strongest for high spin and prograde particle orbits with high eccentricity and low periapsis distance. However, there is no indication that they will completely disappear in any region of the parameter space (although they may become very difficult to separate from the rest of the field due to low amplitudes, high damping rates, and/or longer periods). (3) The effect of aligning the orbital frequencies with the QNM frequencies is very small, and decreases still further when the orbital spectrum becomes denser for more eccentric orbits.

\section{DISCUSSION AND CONCLUSIONS}

In this paper, we study an interesting class of features first observed in the scalar self-force for point particles in orbit in Kerr spacetime [8]. That study identified the feature, introduced the term "wiggles", and argued that it was in some (un- 


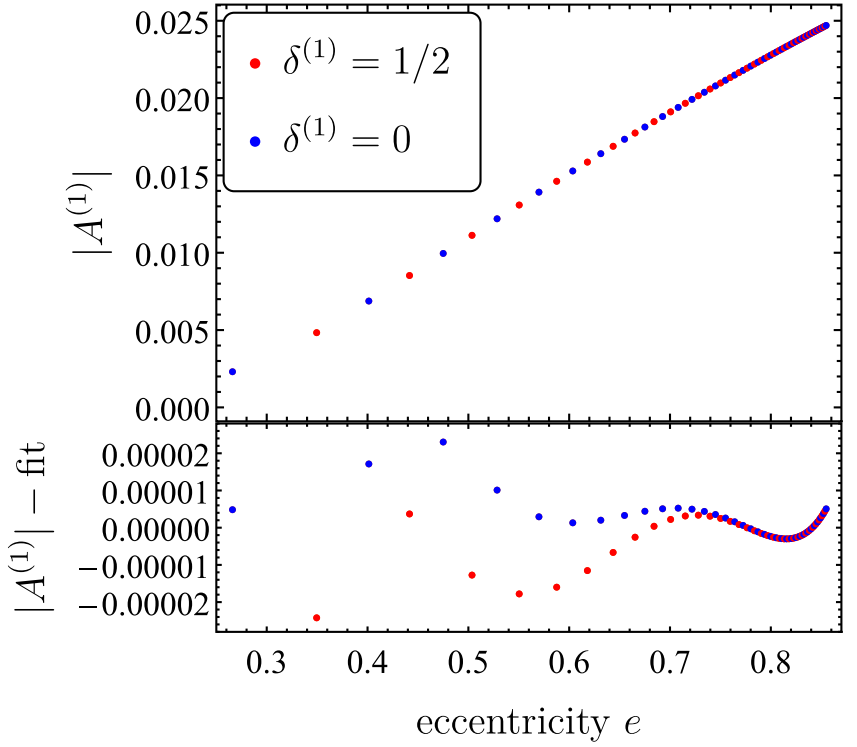

FIG. 12. Dependence of the the fitted QNM amplitude $\left|A_{1}\right|$ of the lowest damped $\mathfrak{l}=m=2 \mathrm{QNM}$ as a function of the eccentricity $e$ for a sequence of orbits with fixed primary spin $a=0.95 \mathrm{M}$ and periapsis distance $r_{\min }=1.85 M$. The parameter $u_{\text {ref }}$ is fixed to coincide with the particle passing through periapsis. The data points are shaded according to the degree of alignment $\delta$ of the particle spectrum with the QNM frequency $\omega^{(1)}$. The lower panel shows the difference between the amplitude and a fitted quintic polynomial in $e$.

specified) manner "caused by the particle's close passage by the large black hole," but did not attempt to attribute it to any particular physical origin. More recently Refs. $[9,10,12]$ have shown further examples of wiggles, demonstrated that wiggle complex frequencies agree with known Kerr quasinormalmode (QNM) frequencies, and concluded that wiggles are in fact "just" a sampling at the measurement point(s) of Kerr QNMs excited by the particle.

Here we survey the phenomenology of wiggles for both the scalar-field and gravitational cases, across a range of Kerr spins and particle orbits. In both the scalar-field and gravitational cases, we find that wiggles are essentially a generic phenomenon, i.e., they occur over a wide range of configuration space without any "fine-tuning" of parameters. Wiggles are observable in field perturbations at fixed spatial positions, in the radiation-reaction "self-force," and in the radiated fields at $\mathcal{J}^{+}$.

In both the scalar-field and gravitational cases, we find that at all observed locations in spacetime, wiggles can be quantitatively fit by models of QNMs sampled at the observation points. In particular, in both the scalar-field and gravitational cases, our fitted wiggle frequencies agree well [in both real (oscillatory) and imaginary (damping) parts] with Kerr QNM frequencies calculated by Berti, Cardoso, and Starinets [69,70] (Figs. 6-8 and Table V).

The appearance of pronounced wiggles appears to rely on three key aspects of the configuration of the system: (1) a highly spinning central (Kerr) black hole (the closer to $\tilde{a}=1$, the more pronounced the effect); (2) a highly eccentric prograde orbit for the particle (the closer to $e=1$, the more pronounced the effect); and (3) a close periapsis passage by

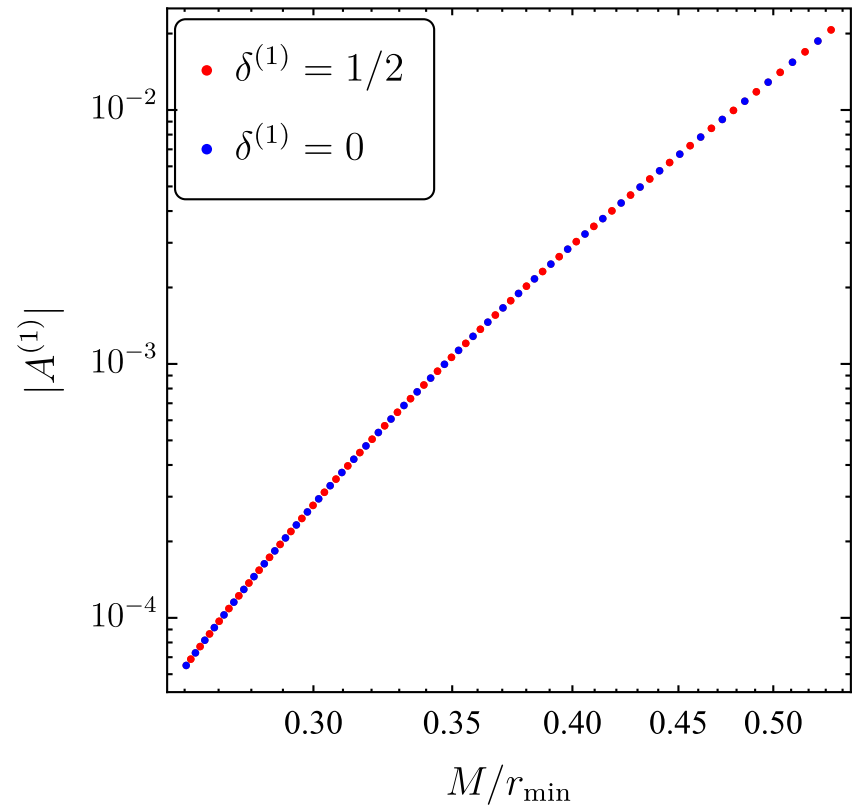

FIG. 13. Dependence of the the fitted QNM amplitude $\left|A_{1}\right|$ of the lowest damped $\mathfrak{l}=m=2$ QNM as a function of the inverse periapsis distance $M / r_{\min }$ for a sequence of orbits with fixed primary spin $a=0.95 M$ and eccentricity $e=0.8$. The parameter $u_{\text {ref }}$ is fixed to coincide with the particle passing through periapsis. The data points are shaded according to the degree of alignment $\delta$ of the particle spectrum with the QNM frequency $\omega^{(1)}$.

the particle (the closer to the light ring, the more pronounced the effect). ${ }^{10}$

This is not surprising: highly spinning black holes have much longer-lived QNMs than those with low spin. Increasing eccentricity of the particle orbit does three things: it increases the strength of the perturbation at the periapsis, it widens the frequency spectrum of the perturbation (increasing the overlap with the QNMs), and it provides a natural "quiet" period when the particle approaches its apoapsis, during which the QNMs can more easily be observed. Finally, bringing the particle periapsis closer to the light ring allows the perturbation to deposit more energy in the QNMs. (QNMs in Kerr spacetime are readily excited by orbits near the light ring [69,72,73].)

Interestingly, we find that the amplitude with which wiggles are excited does not depend sensitively on the particle precise orbital motion near periapsis. Notably, we find that the wiggle amplitude varies smoothly and monotonically with the particle periapsis radius and orbital eccentricity (and with the Kerr spin).

We have not attempted to carefully delineate the exact boundaries of the region in configuration space where wiggles occur (even assuming that there are, in fact, configurations with no QNM excitation, which is not obvious). It is likely that different modeling/fitting schemes could observe and fit low-amplitude and/or rapidly damped wiggles even in some cases where we fail to observe them (e.g., the $\times$ cases in

\footnotetext{
${ }^{10} \mathrm{We}$ refer here to the prograde light ring; we observe only very small QNM excitation when the particle periapsis is close to the retrograde light ring.
} 
Table II). For example, Fig. 11 strongly suggests that although the wiggle amplitude is very small in some cases, wiggles are present for all Kerr spins along this sequence of orbits, including retrograde as well as prograde orbits.

Our scalar-field wiggle modeling/fitting scheme is (deliberately) quite conservative in requiring visual observation of a wiggle in a time-series plot of the original diagnostic. This requirement reduces the risk of false positives (where we would misidentify a fitting or background-spline artifact as a wiggle), at the cost of reducing our sensitivity to lowamplitude and/or rapidly damped wiggles.

An interesting example of these factors at play is the $(\tilde{a}, p, e)=(0.99,8,0.8)$ scalar-field configuration, for which Nasipak, Osburn, and Evans [12] observed and fitted $\ell=m=1, \ell=m=2, \ell=m=3$, and $\ell=m=4$ wiggle (QNM) modes. Their Figs. 8 and 9 show the $\ell=m=4$ wiggle as having an amplitude approximately $10^{9}$ times smaller than the $\ell=m=1$ wiggle; this is only detectable by virtue of the high accuracy and low numerical noise level of their frequency-domain code. In contrast, for this configuration, we observed wiggles for $m=1$ but not for $m \geqslant 2$; this is likely because even the $m=2$ wiggles are already too low in amplitude to be visually observable in the original time series.

Existing astrophysical models of extreme mass ratio binaries [74,75] and observations of highly spinning black holes [76] suggest that it is quite reasonable to expect some fraction of EMRIs to fall within the region of parameter space where wiggles are excited with significant amplitude. (Both the magnitude of this fraction and the absolute numbers of such systems are still very uncertain.)

Given that the QNM excitations appear not just in the local self-force, but also in the gravitational waveform, a natural question is whether they could be experimentally observed by LISA or other detectors. While this is certainly possible in principle, there are two considerations which make it less likely in practice. Most importantly, the effect is quite weak in all but the most extreme cases. In most of the gravitationalfield cases investigated here, it was necessary to zoom in on plots in order to see the wiggles visually, reflecting the fact that their magnitude represents at most a few percent of the total signal. A second consideration in terms of detectability is that the dynamical evolution of EMRIs may tend to avoid the wiggles region of parameter space (e.g., if most EMRIs evolve to low orbital eccentricities while still at relatively large periapsis radii). This would imply that the event rate for detectable EMRI wiggles would be quite low.

Despite these concerns, it would be worthwhile to conduct a more thorough study to quantitatively address the question of detectability of QNM wiggles by LISA or future nextgeneration gravitational-wave detectors. It may even be the case that advanced data analysis techniques could be used to boost the detectability. For example, although an individual wiggle is weak, it will repeat for each orbit throughout the entire inspiral. As noted by Ref. [12], wiggles will appear with almost the same frequency throughout the inspiral (the QNM frequency only depends on the mass and spin parameters of the larger black hole, and these change very little during the inspiral). Moreover, this frequency is much higher than than the main orbital frequency, potentially making it easier to separate these signal components in data analysis.

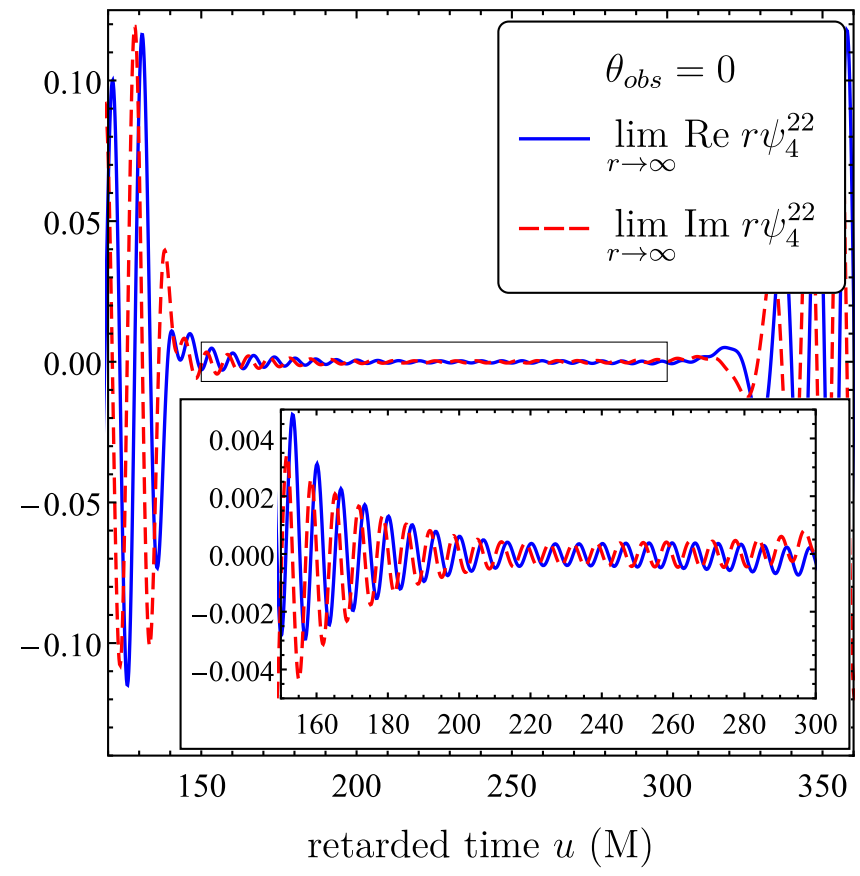

FIG. 14. The $\mathfrak{l}=m=2$ mode of the gravitational waveform at $\mathcal{J}^{+}$for the configuration $(\tilde{a}, p, e)=(0.99999,2.918,0.807)$. The observed wiggles are surprising because the real part of the frequency (in the highlighted area) lies between $0.93 \mathrm{M}^{-1}$ and $0.98 \mathrm{M}^{-1}$, whereas the QNM frequencies are bunched up near $0.995 M^{-1}$.

The analysis done here has been somewhat post-hoc, in that we first identified a feature in the signal and then fit this feature to a damped sinusoid representing a QNM ringdown. Our intuitive interpretation of this QNM ringdown is that it is a result of strong QNM excitation near periapsis, which is then encountered over an extended period later in the orbit. The self-force in curved space-times arises from nonlocal interactions of the object with its self-field, which was generated in the object's past and scattered off the spacetime curvature. The association of wiggles with QNM excitations suggest that they represent a situation where this nonlocal nature of the selfforce is particularly apparent. To more explicitly develop this interpretation, it may be informative to attempt the analysis in the other direction, first by starting with a model for a QNM excitation from a burst of radiation generated near a periapsis passage, and then comparing such a model to the observed signal. This approach would allow one to pinpoint where in the orbit the QNM excitation occurs, would give a deeper understanding of the effect, and may even provide a link to geometric features such as caustics and the propagation of waves on black hole spacetimes. A Green function approach $[77,78]$ would be a natural choice for such a study, but is quite distinct from the methodology used in this paper so we leave it for future work.

In this work, we have focused mostly on systems with somewhat realistic Kerr spins $J / M^{2} \lesssim 0.999$. Initial investigations of the near-extremal regime suggest a rich phenomenology, involving many different QNMs at fixed $\mathfrak{l}$ and $m$. One puzzling result is the $\mathfrak{l}=m=2$ mode generated at $\mathcal{J}^{+}$by a particle orbiting a black hole with spin $J / M^{2}=0.99999$ and the same orbital frequencies as the $(\tilde{a}, p, e)=(0.99,3,0.8)$ 
orbit, shown in Fig. 14. One of the puzzling aspects of this waveform is that the decaying wiggles in the highlighted area have a frequency between $0.93 M^{-1}$ and $0.98 M^{-1}$ (depending on where it is measured), while the nearest QNMs all have frequency close to $0.995 \mathrm{M}^{-1}$. Whether this is the result of some complicated collective behavior of the QNMs or some new physical effect is currently unclear, and should be investigated in future works. ${ }^{11}$

\section{ACKNOWLEDGMENTS}

We thank Leor Barack for invaluable discussions throughout the course of this research. We thank Richard Brito for

\footnotetext{
${ }^{11}$ While we were making final revisions to this manuscript Rifat, Khanna, and Burko [13] reported a detailed study of wiggles in near-extremal Kerr spacetimes, particularly the $(\tilde{a}, p, e)=$ $(0.99999,2.918,0.807)$ system. Their results are consistent with ours; they find that the anomalous wiggle frequencies are an intermediate-time effect caused by the superposition of many simultaneously excited Kerr QNMs.
}

useful discussions about QNMs. We also thank Dan Kennefick, Scott Hughes, Marc Casals, Peter Zimmerman, Conor O'Toole, and Adrian Ottewill for useful discussions on the results of this paper. J.T. thanks the Alexander von Humboldt Foundation for fellowship funding for my stay at the Max-Planck-Institut für Gravitationsphysik (Albert-EinsteinInstitute), and the AEI (Division of Astrophysical and Cosmological Relativity) and Indiana University (Office of the Vice Provost for Research, Center for Spacetime Symmetries, and Department of Astronomy) for additional funding. M.v.d.M. was supported by European Union's Horizon 2020 research and innovation programme under grant agreement No. 705229. Some of the numerical results in this paper were obtained using the IRIDIS High Performance Computing Facility at the University of Southampton and the Karst and Data Capacitor facilities at Indiana University (supported by the U.S. National Science Foundation under Grant No. CNS0521433, by Lilly Endowment, Inc. through its support for the IU Pervasive Technology Institute, and by the Indiana Metabolomics and Cytomics (METACyt) Initiative).
[1] J. R. Gair, L. Barack, T. Creighton, C. Cutler, S. L. Larson, E. S. Phinney, and M. Vallisneri, Event rate estimates for LISA extreme mass ratio capture sources, Class. Quantum Grav. 21, S1595 (2004).

[2] L. Barack and C. Cutler, LISA capture sources: Approximate waveforms, signal-to-noise ratios and parameter estimation accuracy, Phys. Rev. D 69, 082005 (2004).

[3] P. Amaro-Seoane, J. R. Gair, M. Freitag, M. C. Miller, I. Mandel, C. J. Cutler, and S. Babak, Intermediate and extreme mass-ratio inspirals-astrophysics, science applications and detection using LISA, Class. Quantum Grav. 24, R113 (2007).

[4] J. R Gair, Probing black holes at low redshift using LISA EMRI observations, Class. Quantum Grav. 26, 094034 (2009).

[5] Y. Kojima and T. Nakamura, Gravitational radiation from a particle scattered by a Kerr black hole, Prog. Theor. Phys. 72, 494 (1984)

[6] L. M. Burko and G. Khanna, Accurate time-domain gravitational waveforms for extreme-mass-ratio binaries, Europhys. Lett. 78, 60005 (2007)

[7] S. O'Sullivan and S. A. Hughes, Strong-field tidal distortions of rotating black holes: II. Horizon dynamics from eccentric and inclined orbits, Phys. Rev. D 94, 044057 (2016).

[8] J. Thornburg and B. Wardell, Scalar self-force for highly eccentric equatorial orbits in Kerr spacetime, Phys. Rev. D 95, 084043 (2017).

[9] J. Thornburg, presentation at the 19th Capra Meeting on Radiation Reaction, Observatoire de Paris, Meudon, France (2016).

[10] J. Thornburg, presentation at the 20th Capra Meeting on Radiation Reaction, University of North Carolina, Chapel Hill, USA (2017).

[11] H. Yang, W. E. East, V. Paschalidis, F. Pretorius, and R. F. P. Mendes, Evolution of highly eccentric binary neutron stars including tidal effects, Phys. Rev. D 98, 044007 (2018).
[12] Z. Nasipak, T. Osburn, and C. R. Evans, Repeated faint quasinormal bursts in extreme-mass-ratio inspiral waveforms: Evidence from frequency-domain scalar self-force calculations on generic Kerr orbits, Phys. Rev. D 100, 064008 (2019).

[13] N. E.M. Rifat, G. Khanna, and L. M. Burko, Repeated ringing of black holes: Quasinormal bursts from highly eccentric, extreme mass-ratio binaries, Phys. Rev. Research 1, 033150 (2019).

[14] T. Damour, The problem of motion in Newtonian and Einsteinian gravity, in Three Hundred Years of Gravitation, edited by S. W. Hawking and W. Israel (Cambridge University Press, Cambridge, England, 1987), Chap. 6, pp. 128-198.

[15] E. Poisson and C. M. Will, Gravity: Newtonian, Post-Newtonian, Relativistic (Cambridge University Press, Cambridge, England, 2014).

[16] C. M. Will, Theory and Experiment in Gravitational Physics, 2nd ed. (Cambridge University Press, Cambridge, England, 2018).

[17] L. Blanchet, Gravitational radiation from post-Newtonian sources and inspiralling compact binaries, Liv. Rev. Relativ. 17, 2 (2014).

[18] T. Futamase and Y. Itoh, The post-Newtonian approximation for relativistic compact binaries, Liv. Rev. Relat. 10, 2 (2007).

[19] L. Blanchet, Post-Newtonian theory and the two-body problem, in Mass and Motion in General Relativity, edited by L. Blanchet, A. Spallicci, and B. F. Whiting, Fundamental Theories of Physics Vol. 162 (Springer-Verlag, Berlin, 2011), pp. 125-166.

[20] G. Schäfer, Post-Newtonian methods: Analytic results on the binary problem, in Mass and Motion in General Relativity, edited by L. Blanchet, A. Spallicci, and B. F. Whiting, Fundamental Theories of Physics Vol. 162 (Springer-Verlag, Berlin, 2011), pp. 167-210. 
[21] F. Pretorius, Binary black hole coalescence, in Relativistic Objects in Compact Binaries: From Birth to Coalescence, edited by M. Colpi (Springer-Verlag, Berlin, 2007).

[22] M. Hannam, S. Husa, J. G. Baker, M. Boyle, B. Brügmann, T. Chu, N. Dorband, F. Herrmann, I. Hinder, B. J. Kelly, L. E. Kidder, P. Laguna, K. D. Matthews, J. R. van Meter, H. P. Pfeiffer, D. Pollney, C. Reisswig, M. A. Scheel, and D. Shoemaker, Samurai project: Verifying the consistency of black-hole-binary waveforms for gravitational-wave detection, Phys. Rev. D 79, 084025 (2009).

[23] M. Hannam, Status of black-hole-binary simulations for gravitational-wave detection, Class. Quantum Grav. 26, 114001 (2009).

[24] M. Hannam and I. Hawke, Numerical relativity simulations in the era of the Einstein telescope, Gen. Relativ. Gravit. 43, 465 (2011).

[25] M. Campanelli, C. O. Lousto, B. C. Mundim, H. Nakano, Y. Zlochower, and H.-P. Bischof, Advances in simulations of generic black-hole binaries, Class. Quantum Grav. 27, 084034 (2010).

[26] N. T. Bishop, R. Gómez, S. Husa, L. Lehner, and J. Winicour, Numerical relativistic model of a massive particle in orbit near a Schwarzschild black hole, Phys. Rev. D 68, 084015 (2003).

[27] N. T. Bishop, R. Gómez, L. Lehner, M. Maharaj, and J. Winicour, Characteristic initial data for a star orbiting a black hole, Phys. Rev. D 72, 024002 (2005).

[28] C. F. Sopuerta, P. Sun, P. Laguna, and J. Xu, A toy model for testing finite element methods to simulate extreme-mass-ratio binary systems, Class. Quantum Grav. 23, 251 (2006).

[29] C. F. Sopuerta and P. Laguna, Finite element computation of the gravitational radiation emitted by a pointlike object orbiting a nonrotating black hole, Phys. Rev. D 73, 044028 (2006).

[30] C. O. Lousto, H. Nakano, Y. Zlochower, and M. Campanelli, Intermediate-Mass-Ratio Black-Hole Binaries: Numerical Relativity Meets Perturbation Theory, Phys. Rev. Lett. 104, 211101 (2010).

[31] C. O. Lousto and Y. Zlochower, Orbital Evolution of ExtremeMass-Ratio Black-Hole Binaries with Numerical Relativity, Phys. Rev. Lett. 106, 041101 (2011).

[32] S. Husa, S. Khan, M. Hannam, M. Pürrer, F. Ohme, X. J. Forteza, and A. Bohé, Frequency-domain gravitational waves from nonprecessing black-hole binaries. I. New numerical waveforms and anatomy of the signal, Phys. Rev. D 93, 044006 (2016).

[33] I. Hinder (personal communication).

[34] B. Wardell, I. Vega, J. Thornburg, and P. Diener, Generic effective source for scalar self-force calculations, Phys. Rev. D 85, 104044 (2012).

[35] M. van de Meent and A. G. Shah, Metric perturbations produced by eccentric equatorial orbits around a Kerr black hole, Phys. Rev. D 92, 064025 (2015).

[36] M. van de Meent, Gravitational self-force on eccentric equatorial orbits around a Kerr black hole, Phys. Rev. D 94, 044034 (2016).

[37] M. van de Meent, Self-Force Corrections to the Periapsis Advance Around a Spinning Black Hole, Phys. Rev. Lett. 118, 011101 (2017).

[38] M. van de Meent, Gravitational self-force on generic bound geodesics in Kerr spacetime, Phys. Rev. D 97, 104033 (2018).
[39] R. M. Wald, General Relativity (The University of Chicago Press, Chicago, 1984).

[40] D. R. Brill, P. L. Chrzanowski, C. M. Pereira, E. D. Fackerell, and J. R. Ipser, Solution of the scalar wave equation in a Kerr background by separation of variables, Phys. Rev. D 5, 1913 (1972).

[41] S. A. Teukolsky, Perturbations of a rotating black hole. I. Fundamental equations for gravitational, electromagnetic, and neutrino-field perturbations, Astrophys. J. 185, 635 (1973).

[42] P. A. Sundararajan, G. Khanna, and S. A. Hughes, Towards adiabatic waveforms for inspiral into Kerr black holes: A new model of the source for the time domain perturbation equation, Phys. Rev. D 76, 104005 (2007).

[43] L. Barack and D. A. Golbourn, Scalar-field perturbations from a particle orbiting a black hole using numerical evolution in $2+1$ dimensions, Phys. Rev. D 76, 044020 (2007).

[44] I. Vega and S. Detweiler, Regularization of fields for self-force problems in curved spacetime: Foundations and a time-domain application, Phys. Rev. D 77, 084008 (2008).

[45] I. Vega, B. Wardell, and P. Diener, Effective source approach to self-force calculation, Class. Quantum Grav. 28, 134010 (2011).

[46] S. Detweiler and B. F. Whiting, Self-force via a Green's function decomposition, Phys. Rev. D 67, 024025 (2003).

[47] A. Zenginoğlu, Hyperboloidal foliations and scri-fixing, Class. Quantum Grav. 25, 145002 (2008).

[48] A. Zenginoğlu, Hyperboloidal evolution with the Einstein equations, Class. Quantum Grav. 25, 195025 (2008).

[49] A. Zenginoğlu, Hyperboloidal layers for hyperbolic equations on unbounded domains, J. Comp. Phys. 230, 2286 (2011).

[50] A. Zenginoğlu and G. Khanna, Null Infinity Waveforms from Extreme-Mass-Ratio Inspirals in Kerr Spacetime, Phys. Rev. X 1, 021017 (2011).

[51] A. Zenginoğlu and L. E. Kidder, Hyperboloidal evolution of test fields in three spatial dimensions, Phys. Rev. D 81, 124010 (2010).

[52] A. Zenginoğlu and M. Tiglio, Spacelike matching to null infinity, Phys. Rev. D 80, 024044 (2009).

[53] S. Bernuzzi, A. Nagar, and A. Zenginoğlu, Binary black hole coalescence in the large-mass-ratio limit: The hyperboloidal layer method and waveforms at null infinity, Phys. Rev. D 84, 084026 (2011).

[54] S. Bernuzzi, A. Nagar, and A. Zenginoğlu, Horizon-absorption effects in coalescing black-hole binaries: An effective-onebody study of the nonspinning case, Phys. Rev. D 86, 104038 (2012).

[55] S. Mano and E. Takasugi, Analytic solutions of the Teukolsky equation and their properties, Prog. Theor. Phys. 97, 213 (1997).

[56] S. Mano, H. Suzuki, and E. Takasugi, Analytic solutions of the Teukolsky equation and their low frequency expansions, Prog. Theor. Phys. 95, 1079 (1996).

[57] R. M. Wald, On perturbations of a Kerr black hole, J. Math Phys. 14, 1453 (1973).

[58] J. M. Cohen and L. S. Kegeles, Electromagnetic fields in curved spaces-A constructive procedure, Phys. Rev. D 10, 1070 (1974).

[59] L. S. Kegeles and J. M. Cohen, Constructive procedure for perturbations of space-times, Phys. Rev. D 19, 1641 (1979). 
[60] P. L. Chrzanowski, Vector potential and metric perturbations of a rotating black hole, Phys. Rev. D 11, 2042 (1975).

[61] R. M. Wald, Construction of Solutions of Gravitational, Electromagnetic, or Other Perturbation Equations from Solutions of Decoupled Equations, Phys. Rev. Lett. 41, 203 (1978).

[62] L. Barack, A. Ori, and N. Sago, Frequency-domain calculation of the self force: The high-frequency problem and its resolution, Phys. Rev. D 78, 084021 (2008).

[63] L. Barack and A. Ori, Gravitational Self-force on a Particle Orbiting a Kerr Black Hole, Phys. Rev. Lett. 90, 111101 (2003).

[64] L. Barack, Gravitational self force in extreme mass-ratio inspirals, Class. Quantum Grav. 26, 213001 (2009).

[65] A. Pound, C. Merlin, and L. Barack, Gravitational self-force from radiation-gauge metric perturbations, Phys. Rev. D 89, 024009 (2014).

[66] C. Merlin, A. Ori, L. Barack, A. Pound, and M. van de Meent, Completion of metric reconstruction for a particle orbiting a Kerr black hole, Phys. Rev. D 94, 104066 (2016).

[67] M. van de Meent, The mass and angular momentum of reconstructed metric perturbations, Class. Quantum Grav. 34, 124003 (2017).

[68] J. J. Moré, B. S. Garbow, and K. E. Hillstrom, User Guide for MINPACK-1, Tech. Rep. ANL-80-74 (Argonne National Laboratory, Argonne, USA, 1980) available from the NETLIB online software repository, http://www.netlib.org/ minpack/.
[69] E. Berti, V. Cardoso, and A. O Starinets, Quasinormal modes of black holes and black branes, Class. Quantum Grav. 26, 163001 (2009).

[70] E. Berti, V. Cardoso, and C. M. Will, On gravitational-wave spectroscopy of massive black holes with the space interferometer LISA, Phys. Rev. D 73, 064030 (2006).

[71] Data tables downloaded from https://pages.jh.edu/ eberti2/ ringdown/ on 19 April 2019.

[72] C. J. Goebel, Comments on the "vibrations" of a black hole, Astrophys. J. 172, L95 (1972).

[73] G. Khanna and R. H. Price, Black hole ringing, quasinormal modes, and light rings, Phys. Rev. D 95, 081501(R) (2017).

[74] M. C. Miller, T. Alexander, P. Amaro-Seoane, A. J. Barth, C. Cutler, J. R. Gair, C. Hopman, D. Merritt, E. S. Phinney, and D. O. Richstone, Probing stellar dynamics in galactic nuclei, arXiv:0903.0285.

[75] C. Hopman and T. Alexander, The orbital statistics of stellar inspiral and relaxation near a massive black hole: Characterizing gravitational wave sources, Astrophys. J. 629, 362 (2005).

[76] L. Brenneman, Measuring Supermassive Black Hole Spins in Active Galactic Nuclei, SpringerBriefs in Astronomy (SpringerVerlag, New York, 2013).

[77] M. Casals, S. Dolan, A. C. Ottewill, and B. Wardell, Self-force and Green function in Schwarzschild spacetime via quasinormal modes and branch cut, Phys. Rev. D 88, 044022 (2013).

[78] B. Wardell, C. R. Galley, A. Zenginoğlu, M. Casals, S. R. Dolan, and A. C. Ottewill, Self-force via Green functions and worldline integration, Phys. Rev. D 89, 084021 (2014). 